\title{
Evaluation of the Economic Relationships on the Basis of Statistical Decision-Making in Complex Neutrosophic Environment
}

\author{
Abdul Nasir, ${ }^{1}$ Naeem Jan $\mathbb{D}^{1},{ }^{1}$ Abdu Gumaei $\mathbb{D}^{2},{ }^{2}$ Sami Ullah Khan, ${ }^{1}$ \\ and Mabrook Al-Rakhami iD ${ }^{3}$ \\ ${ }^{1}$ Department of Mathematics, Institute of Numerical Sciences, Gomal University, Dera Ismail Khan 29050, KPK, Pakistan \\ ${ }^{2}$ Computer Science Department, Faculty of Applied Sciences, Taiz University, Taiz 6803, Yemen \\ ${ }^{3}$ Research Chair of Pervasive and Mobile Computing, Department of Information Systems, \\ College of Computer and Information Sciences, King Saud University, Riyadh 11543, Saudi Arabia
}

Correspondence should be addressed to Abdu Gumaei; abdugumaei@gmail.com and Mabrook Al-Rakhami;

malrakhami@ksu.edu.sa

Received 22 February 2021; Revised 7 April 2021; Accepted 12 April 2021; Published 3 May 2021

Academic Editor: Ahmed Mostafa Khalil

Copyright $\odot 2021$ Abdul Nasir et al. This is an open access article distributed under the Creative Commons Attribution License, which permits unrestricted use, distribution, and reproduction in any medium, provided the original work is properly cited.

\begin{abstract}
Fuzzy sets and fuzzy logics are used to model events with imprecise, incomplete, and uncertain information. Researchers have developed numerous methods and techniques to cope with fuzziness or uncertainty. This research intends to introduce the novel concepts of complex neutrosophic relations (CNRs) and its types based on the idea of complex neutrosophic sets (CNSs). In addition, these concepts are supported by suitable examples. A CNR discusses the quality of a relationship using the degree of membership, the degree of abstinence, and the degree of nonmembership. Each of these degrees is a complex number from the unit circle in a complex plane. The real part of complex-valued degrees represents the amplitude term, while the imaginary part represents the phase term. This property empowers CNRs to model multidimensional variables. Moreover, some interesting properties and useful results have also been proved. Furthermore, the practicality of the proposed concepts is verified by an application, which discusses the use of the proposed concepts in statistical decision-making. Additionally, a comparative analysis between the novel concepts of CNRs and the existing methods is carried out.
\end{abstract}

\section{Introduction}

In mathematics, the word modeling refers to the process of representing real-world events in a mathematical form. There are many ways to express the practical happenings in the mathematical form which depend on the nature of the problem. In real life, there are many occasions when one faces uncertainty, vagueness, and ambiguity. Fuzzy sets and logics introduced by Zadeh [1] are proved to be great tools at dealing with problems that involve doubts, vagueness, and imprecise information. Fuzzy sets (FSs) are characterized by a mapping called the degree of membership $(\mathfrak{m})$ that attains real values from 0 to 1 , like probability. Atanassov [2] developed the idea of intuitionistic fuzzy sets (IFSs) that also model fuzziness. The advancement in IFSs as compared to FSs is that IFSs discuss the degree of membership $(\mathfrak{m})$ as well the degree of nonmembership ( $\mathfrak{n}$ ) of the events. Both degrees attain values from the unit interval provided that their sum is contained within the unit interval. Due to this constraint on the sum, a decision maker is bounded and limited in assigning the values to degrees of membership and nonmembership. For instance, a decision maker cannot assign $\mathfrak{m}=0.8$ and $\mathfrak{n}=0.5$ because their sum exceeds 1 . This limitation affects the precision of the results. Henceforth, Yager [3] provided the notion of Pythagorean fuzzy sets (PFSs). A PFS is a generalization of IFS and FS that eases the constraints in IFS. Like IFSs, the PFSs also discuss the degree of membership $(\mathfrak{m})$ and the degree of nonmembership $(\mathfrak{n})$ 
that are fuzzy numbers, provided that the sum $\mathfrak{m}^{2}+\mathfrak{n}^{2}$ belongs to the unit interval. Although PFS provides a broader range of fuzzy numbers to be assigned as degrees as compared to an IFS, there are instances when someone needs to set both the degrees higher enough so that the sum disobeys the restrictions of PFSs. For example if $\mathfrak{m}=0.9$ and $\mathfrak{n}=0.7$, then $0.9^{2}+0.7^{2}=0.81+0.49 \not 1$. Keeping this in mind, Yager [4] generalized IFSs and PFSs to devise the notion of q-rung orthopair fuzzy sets (qROFSs). A qROFS is one of the most powerful tools to tackle fuzziness when discussing the degree of membership and the degree of nonmembership. According to qROFSs, both the degrees $\mathfrak{m}$ and $\mathfrak{n}$ are fuzzy numbers and $0 \leq \mathfrak{m}^{q}+\mathfrak{n}^{q} \leq 1$, where $q$ is a positive integer. For $q=1$ and $q=2$, the qROFS transforms to an IFS and PFS, respectively. Garg [5] presented applications of PFSs in multiattribute decision-making process. Yang and Hussain $[6,7]$ introduced fuzzy entropy, distance, and similarity measures of PFSs with applications to multicriteria decision-making. Zhou et al. [8] also introduced divergence measure of PFSs and applied them in medical diagnosis. Yang et al. [9] gave the idea of belief and plausibility measures on IFSs with construction of belief-plausibility TOPSIS. Using the characteristic objects method, Faizi et al. [10] proposed IFSs in multicriteria group decision-making problems. Peng and Liu [11] devised information measures for qROFSs. Wei et al. [12] initiated the concept of qROF Heronian mean operators in multiple attribute decision-making, and Liu et al. [13] developed some cosine similarity measures and distance measures between q-rung orthopair fuzzy sets.

Later, Smarandache [14] introduced neutrosophic sets (NSs) that are the generalization of FSs. In an NS, there are three independent fuzzy-valued mappings, i.e., the degree of membership $(\mathfrak{m})$, the degree of abstinence $(\mathfrak{a})$, and the degree of nonmembership $(\mathfrak{n})$. According to the NSs, the condition on the sum of the degrees is that $0 \leq \mathfrak{m}+\mathfrak{a}+\mathfrak{n} \leq 3$. This theory permits the decision makers to freely assign any fuzzy value to an object as its degrees of membership, abstinence, and nonmembership. Wang et al. [15] devised single-valued NSs (SVNSs), Smarandache [16-20] scrupulously researched the NSs and provided several generalizations of NSs, Salama and Alblowi [21] worked on NS and neutrosophic topological spaces, Das et al. [22] applied the NS in decision-making, Khalil et al. [23] gave the combination of the SVNSs and their application in decisionmaking, and Sahin and Liu [24] presented the correlation coefficient of SVN hesitant FSs and applied them in decision-making. Hashim et al. [25] defined and applied the concept of neutrosophic bipolar fuzzy set in the preparation of medicines.

An idea of involving the complex numbers in the FS theory lead to the development of a new idea; complex FS (CFS) which was concocted by Ramot et al. [26]. A CFS is characterized by a complex-valued mapping, called the degree of membership $\left(\mathfrak{m}_{\mathbb{C}}\right)$. The degree of membership $\left(\mathfrak{m}_{\mathbb{C}}\right)$ acquires values from the unit circle in a complex plane. For an object $\mathrm{n}$, the degree of membership is defined as $\mathfrak{m}_{\mathbb{C}}(x)=\tau_{\mathbb{C}}(x) e^{\rho_{\mathbb{C}}(x) 2 \pi i}$, where $\tau_{\mathbb{C}}$ and $\rho_{\mathbb{C}}$ are fuzzy numbers and are known as the amplitude term and the phase term, respectively. The preeminence of CFSs over FSs is that CFSs are capable of modeling multidimensional problems. The phase term usually refers to time. Alkouri et al. [27] presented the concept of complex IFSs (CIFSs) that characterizes an object with a pair of complex-valued mappings, i.e., degrees of membership $\mathfrak{m}_{\mathbb{C}}$ and nonmembership $\mathfrak{n}_{\mathbb{C}}$. Both the degrees belong to the unit circle in a complex plane and so does their sum. Equivalently, the amplitude and phase terms of both the degrees, the sum of amplitude terms, and the sum of phase terms are all fuzzy numbers. Moreover, Ullah et al. [28] introduced the concept of complex PFS (CPFS) that discusses the degree of membership and nonmembership. These degrees are complex numbers from a unit circle in complex plane provided that the sum of their squares is also a complex number in a unit circle. Furthermore, the CIFSs and CPFSs were generalized to complex qROFSs (CqROFSs) by Liu et al. [29] by updating the constraints on the sum of the degrees of membership and nonmembership. According to CqROFSs, the degree of membership $\left(\mathfrak{m}_{\mathbb{C}}\right)$, the degree of nonmembership $\left(\mathfrak{n}_{\mathbb{C}}\right)$, and the sum $\left(\mathfrak{m}_{\mathbb{C}}\right)^{q}+\left(\mathfrak{n}_{\mathbb{C}}\right)^{q}$ lie in a unit circle in a complex plane. Bi et al. [30] defined CF arithmetic aggregation operators, and Tamir et al. [31] presented an overview of theory and applications of CFSs and CF logic. Also, Tamir and Kandel [32] presented the axiomatic theory of CF logic and classes. Ma et al. [33] proposed the method of applying CFSs in multiple periodic factor prediction problems. Ngan et al. [34] generalized the CIFSs by space of quaternion numbers, Garg and Rani [35] offered the coefficient measure of CIFSs and their applications in decision-making, and Rani and Garg [36] introduced the CIF power aggregation operators and applied them in decision-making. Ali and Mahmood [37] gave the idea of Maclaurin symmetric mean operators for CqROFSs and presented their applications. Liu et al. [38] extended the prioritized weighted aggregation operators for decision-making under CqROFSs.

In addition, complex NSs (CNSs) were proposed by Ali and Smarandache [39]. A CNS is characterized by three complex-valued mapping, i.e., degree of membership $\left(\mathfrak{m}_{\mathbb{C}}\right)$, degree of abstinence $\left(\mathfrak{a}_{\mathbb{C}}\right)$, and degree of nonmembership $\left(\mathfrak{n}_{\mathbb{C}}\right)$, such that each of these degrees is a fuzzy number, and their sum is restricted as $0 \leq\left|\mathfrak{m}_{\mathbb{C}}\right|+\left|\mathfrak{a}_{\mathbb{C}}\right|+\left|\mathfrak{n}_{\mathbb{C}}\right| \leq 3$. Note that every complex-valued degree consists of two terms. Each of these terms is a fuzzy number representing two different entities. The advantage of CNSs over other CFSs and its generalizations is that CNSs discuss three independent degrees instead of two. Furthermore, it provides much more freedom to a decision maker because he/she can choose independently any value for each degree from $[0,1]$. Broumi et al. [40] discussed the bipolar CNSs with applications. Furthermore, Gulistan et al. [41] introduced the CN subsemigroups and ideals. Ali and Mahmood [42], Al-Quran and Hassan [43], Manna et al. [44], and Dat et al. [45] applied the CNSs for decision-making, and Singh [46] used CNSs to analyze the air quality.

Klir and Folger [47] presented the concept of crisp relations (CRs) that are based on the crisp set theory. CRs describe the existence of a relationship between some events. Mendel [48] gave the concept of fuzzy relations (FRs), which 
are the extension of CRs. Like its predecessor, FRs also describe the existence of the relationship among the objects, but in addition, FRs also indicate the strength of the relationship by the degree of membership. If the value of degree of membership is nearer to 0 , then it means the relationship is weak, and the value closer to 1 indicates the stronger relationship. For instance, a relationship with the degree of membership 0.5 is weaker than the relationship with the degree 0.6. Moreover, the notion of intuitionistic FRs (IFRs) was introduced by Burillo and Bustince [49]. IFRs describe the quality of relationship by degree of membership and degree of nonmembership, provided that their sum does not exceed 1. Ramot et al. [26] devised the notion of complex FR (CFR) which discusses the complex-valued degree of membership. Ejegwa [50] improved the composition relation for PFSs and applied the concept in medical diagnosis. Ramot et al. [51] worked on CF logic. Hu et al. [52] discovered the distances of CFSs and continuity of CF operations. Deschrijver and Kerre [53] worked on the composition of IFRs, Bustince and Burillo [54] studied the structures of the IFRs, Li et al. [55] proposed some preference relations based on qROFSs, and Zhang et al. [56] offered the concepts of additive consistency-based prioritygenerating method of qROF preference relation.

This paper aims to introduce the notion of complex NRs (CNRs) and its types such as inverse CNR, CN reflexive, irreflexive, symmetric, asymmetric, antisymmetric, transitive, composite, equivalence, order relations, and $\mathrm{CN}$ equivalence class. Besides these, some interesting properties and useful results have also been proved. Since CNRs carry three degrees, i.e., the degrees of membership, abstinence, and nonmembership, they define the quality of a relationship much efficiently. The complex degrees consist of two parts that are the amplitude and phase terms as discussed earlier, so CNRs are capable of describing the problems with time periods, phase changes or multidimensions. An application is also presented to illuminate the practicality of the proposed concepts. The application discusses the worth of the proposed work for a statistician who is supposed to make the decision for the economic policy. In the process of policy making, the data are collected, organized, and analyzed through statistical techniques such as percentages, averages, frequencies, and probabilities and then presented in the form of tables and graphs, and finally the interpretation of the information takes place. On large scales, the information is probably ambiguous, uncertain, or unclear that certainly affects the final decision. In order to cope with such issues, this study proposes a new method in the application.

This paper is organized such that Section 2 defines some fundamental concepts. Section 3 proposes the main objectives and results of the study. Application of CNSs and CNRs in investigating the economic relationships through statistical decision-making is presented in Section 4. Section 5 is named comparative analysis which compares the proposed work with the existing methods. Finally, the paper ends with a conclusion.

\section{Preliminaries}

This section defines some fundamental concepts such as FSs, CFSs, Cartesian product of CFSs, CFRs, IFSs, CIFSs, PFSs, CPFSs, qROFSs, CqROFSs, NSs, and CNSs.

Definition 1 (see [1]). A fuzzy set (FS) $\dddot{A}$ of a referential set $\dddot{U}$ is characterized by a real-valued function $\mathfrak{m}: \mathfrak{U} \longrightarrow[0,1]$ known as the degree of membership of $x \in \dddot{A}$.

Definition 2 (see [26]). A complex FS (CFS) $\ddot{A}$ of a referential set $\mathfrak{U}$ is characterized by a complex-valued function $\mathfrak{m}_{\ddot{A}_{\mathbb{C}}}: \dddot{\mathfrak{U}} \longrightarrow\{\approx|\approx \in \mathbb{C},| \approx \mid \leq 1\}$ known as the degree of membership of $x \in \dddot{A}$, where $z(x)=\tau_{\dddot{A}_{C}}(x) e^{\rho_{\ddot{A}_{C}}(x) 2 \pi i}$ and $0 \leq \tau_{\ddot{A}_{\mathbb{C}}}(x), \rho_{\ddot{A}_{\mathbb{C}}}(x) \leq 1$. $\tau_{\mathbb{C}}(x)$ and $\rho_{\mathbb{C}}(x)$ are known as the amplitude and phase terms, respectively.

Definition 3 (see [26]). The Cartesian product of two CFSs $\dddot{A}=\left\{x_{\mathrm{i}}, \mathfrak{m}_{\ddot{A}_{\mathrm{C}}}\left(x_{\mathrm{i}}\right) \mid x_{\mathrm{i}} \in \dddot{\mathfrak{U}}\right\}$ and $\dddot{B}=\left\{x_{j}, \mathfrak{m}_{\ddot{B}_{\mathrm{C}}}\left(x_{j}\right) \mid x_{j} \in \dddot{\mathfrak{U}}\right\}$, $\mathrm{i}, j \in \mathbb{N}$, is given by

$$
\dddot{A} \times \dddot{B}=\left\{\left(x_{\mathrm{i}}, x_{j}\right), \mathfrak{m}_{\left(\dddot{A} \times{ }_{B}\right)_{\mathrm{C}}}\left(x_{\mathrm{i}}, x_{j}\right) \mid x_{\mathrm{i}} \in \dddot{A}, x_{j} \in \dddot{B}\right\} .
$$

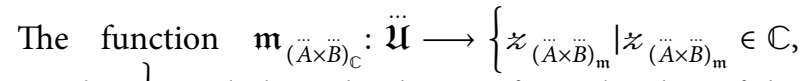
$\left.\left|\approx(\ddot{A} \times \ddot{B})_{\mathfrak{m}}\right| \leq 1\right\}$ symbolizes the degree of membership of the Cartesian product $\dddot{A} \times \dddot{B}$ that is defined as

$$
\mathfrak{m}_{(\ddot{A} \times \ddot{B})_{C}}\left(x_{\mathfrak{i}}, x_{j}\right)=\min \left\{\mathfrak{m}_{\ddot{A}_{\mathbb{C}}}\left(x_{\mathfrak{i}}\right), \mathfrak{m}_{\ddot{B}_{\mathbb{C}}}\left(x_{j}\right)\right\} .
$$

Or equivalently

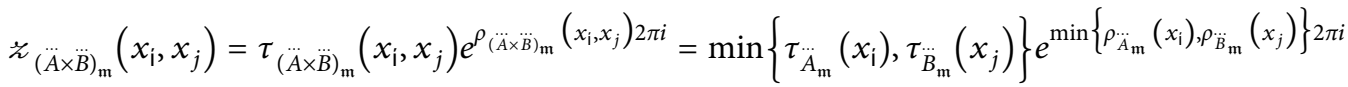

$$
\begin{aligned}
& \cdot \tau_{(\ddot{A} \times \ddot{B})_{\mathrm{m}}}\left(x_{\mathrm{i}}, x_{j}\right), \quad \rho_{(\ddot{A} \times \ddot{B})_{\mathrm{m}}}\left(x_{\mathrm{i}}, x_{j}\right) \in[0,1] .
\end{aligned}
$$


Definition 4 (see [26]). A complex fuzzy relation (CFR) denoted by $\dddot{R}$ is any nonempty subset of $\dddot{A} \times \dddot{B}$, where $\dddot{A}$ and $\dddot{B}$ are CFSs.
Example 1. For given CFS, $\dddot{A}=\left\{\left(x, 0.6 e^{(0.3) 2 \pi i}\right), \quad(y\right.$, $\left.\left.0.3 e^{(0.7) 2 \pi i}\right),\left(\varkappa, 0.9 e^{(0.9) 2 \pi i}\right)\right\}$

The Cartesian product of $\dddot{A}$ to itself is

$$
\dddot{A} \times \dddot{A}=\left\{\begin{array}{c}
\left((x, x), 0.6 e^{(0.3) 2 \pi i}\right),\left((x, y), 0.3 e^{(0.3) 2 \pi i}\right),\left((x, \approx), 0.6 e^{(0.3) 2 \pi i}\right) \\
\left((y, x), 0.3 e^{(0.3) 2 \pi i}\right),\left((y, y), 0.3 e^{(0.7) 2 \pi i}\right),\left((y, \varkappa), 0.3 e^{(0.7) 2 \pi i}\right) \\
\left((\varkappa, x), 0.6 e^{(0.3) 2 \pi i}\right),\left((x, y), 0.3 e^{(0.7) 2 \pi i}\right),\left((\varkappa, \varkappa), 0.9 e^{(0.9) 2 \pi i}\right)
\end{array}\right\},
$$

and the CFR is

$$
\begin{aligned}
\dddot{R}= & \left\{\left((x, y), 0.3 e^{(0.3) 2 \pi i}\right),\left((x, \varkappa), 0.6 e^{(0.3) 2 \pi i}\right),\right. \\
& \left.\cdot\left((y, \varkappa), 0.3 e^{(0.7) 2 \pi i}\right),\left((\varkappa, x), 0.6 e^{(0.3) 2 \pi i}\right)\right\} .
\end{aligned}
$$

Definition 5 (see [2]). An intuitionistic FS (IFS) $\dddot{A}$ of a referential set $\mathfrak{U}$ is characterized by a pair of real-valued functions $\mathfrak{m}, \mathfrak{n}: \dddot{\mathfrak{U}} \longrightarrow[0,1]$ known as the degrees of membership and nonmembership, respectively, of $x \in \dddot{A}$ as long as $0 \leq \mathfrak{m}(x)+\mathfrak{n}(x) \leq 1$.

Definition 6 (see [27]). A complex IFS (CIFS) $\dddot{A}$ of a referential set $\mathfrak{U}$ is characterized by a pair of complex-valued functions $\mathfrak{m}_{\ddot{A}_{\mathbb{C}}}: \dddot{\mathfrak{U}} \longrightarrow\left\{\varkappa_{\mathfrak{m}}\left|\varkappa_{\mathfrak{m}} \in \mathbb{C},\right| \varkappa_{\mathfrak{m}} \mid \leq 1\right\}$ and $\mathfrak{n}_{\ddot{A}_{\mathbb{C}}}: \dddot{\mathfrak{U}}$ $\longrightarrow\left\{\varkappa_{\mathfrak{n}}\left|\varkappa_{\mathfrak{n}} \in \mathbb{C},\right| \varkappa_{\mathfrak{n}} \mid \leq 1\right\}$ known as the degrees of membership and nonmembership, respectively, of $x \in \dddot{A}$ where $\varkappa_{\ddot{A}_{\mathfrak{m}}}(x)=\tau_{\ddot{A}_{\mathfrak{m}}}(x) e^{\rho_{\dddot{A}_{\mathfrak{m}}}(x) 2 \pi i}$ and $\varkappa_{\ddot{A}_{\mathfrak{n}}}(x)=\tau_{\ddot{A}_{\mathfrak{n}}}(x) e^{\rho_{\ddot{A}_{\mathfrak{n}}}(x) 2 \pi i}$. A CIFS has the condition that $0 \leq\left|\mathfrak{m}_{\ddot{A}_{C}}(x)\right|+\left|\mathfrak{n}_{\ddot{A}_{C}}(x)\right| \leq 1$ or equivalently, $\quad 0 \leq \tau_{\dddot{A}_{\mathfrak{m}}}(x)+\tau_{\ddot{A}_{\mathfrak{n}}}(x) \leq 1, \quad 0 \leq \rho_{\ddot{A}_{\mathfrak{m}}}(x)+\rho_{\ddot{A}_{\mathfrak{n}}}(x)$ $\leq 1$., $\tau_{\ddot{A}_{\mathfrak{n}}}(x)$ are known as the amplitude terms, and $\rho_{\ddot{A}_{\mathfrak{m}}}(x)$, $\rho_{\ddot{A}_{n}}(x)$ are known as the phase terms.

Definition 7 (see [4]). A q-rung orthopair FS (qROFS) $\dddot{A}$ of a referential set $\mathfrak{U}$ is characterized by a pair of real-valued functions $\mathfrak{m}, \mathfrak{n}: \dddot{\mathfrak{U}} \longrightarrow[0,1]$ known as the degrees of membership and nonmembership, respectively, of $x \in \dddot{A}$ as long as $0 \leq(\mathfrak{m}(x))^{n}+(\mathfrak{n}(x))^{n} \leq 1$, where $n$ is any natural number.

Note 1 . For $n=1$ and $n=2$, the qROFS converts to an IFS and a Pythagorean fuzzy set (PFS), respectively.

Definition 8 (see [29]). A complex qROFS (CqROFS) $\dddot{A}$ of a referential set $\mathfrak{U}$ is characterized by a pair of complex-valued functions $\mathfrak{m}_{\ddot{A}_{\mathbb{C}}}: \dddot{\mathfrak{U}} \longrightarrow\left\{\varkappa_{\mathfrak{m}}\left|\varkappa_{\mathfrak{m}} \in \mathbb{C},\right| \varkappa_{\mathfrak{m}} \mid \leq 1\right\}$ and $\mathfrak{n}_{\dddot{A}_{\mathbb{C}}}: \dddot{\mathfrak{U}}$ $\longrightarrow\left\{\varkappa_{\mathfrak{n}}\left|\varkappa_{\mathfrak{n}} \in \mathbb{C},\right| \varkappa_{\mathfrak{n}} \mid \leq 1\right\}$ known as the degrees of membership and nonmembership, respectively, of $x \in \dddot{A}$ where
$\varkappa_{\dddot{A}_{\mathfrak{m}}}(x)=\tau_{\dddot{A}_{\mathfrak{m}}}(x) e^{\rho_{\dddot{A}_{\mathfrak{m}}}(x) 2 \pi i}$ and $\varkappa_{\ddot{A}_{\mathfrak{n}}}(x)=\tau_{\dddot{A}_{\mathfrak{n}}}(x) e^{\rho_{\dddot{A}_{\mathfrak{n}}}(x) 2 \pi i} \cdot \mathrm{A}$ CqROFS has the condition that $0 \leq\left|\mathfrak{m}_{\ddot{A}_{\mathbb{C}}}(x)\right|^{n}+\left|\mathfrak{n}_{\ddot{A}_{\mathbb{C}}}(x)\right|^{n}$ $\leq 1$ or, equivalently, $0 \leq\left(\tau_{\dddot{A}_{\mathfrak{m}}}(x)\right)^{n}+\left(\tau_{\dddot{A}_{\mathfrak{n}}}(x)\right)^{n} \leq 1,0 \leq\left(\rho_{\ddot{A}_{\mathfrak{m}}}\right.$ $(x))^{n}+\left(\rho_{A_{\mathfrak{n}}}(x)\right)^{n} \leq 1$, where $n$ is any natural number. $\tau_{\dddot{A}_{\mathfrak{m}}}(x), \tau_{\dddot{A}_{\mathfrak{n}}}(x)$ are known as the amplitude terms, and $\rho_{\ddot{A}_{\mathfrak{m}}}(x), \rho_{\ddot{A}_{\mathfrak{n}}}(x)$ are known as the phase terms.

Note 2. For $n=1$ and $n=2$, the CqROFS converts to $n$ CIFS and a complex PFS (CPFS), respectively.

Definition 9 (see [14]). A neutrosophic set (NS) $\ddot{A}$ of a referential set $\mathfrak{U}$ is characterized by three real-valued functions $\mathfrak{m}, \mathfrak{a}, \mathfrak{n}: \ddot{\mathfrak{U}} \longrightarrow[0,1]$ known as the degrees of membership, abstinence, and nonmembership, respectively, of $x \in \dddot{A}$ as long as $0 \leq \mathfrak{m}(x)+\mathfrak{a}(x)+\mathfrak{n}(x) \leq 3$.

Definition 10 (see [39]). A complex NS (CNS) $\dddot{A}$ of a referential set $\mathfrak{U}$ is characterized by three complex-valued functions $\mathfrak{m}_{\ddot{A}_{\mathbb{C}}}: \dddot{\mathfrak{U}} \longrightarrow\left\{\varkappa_{\dddot{A}_{\mathfrak{m}}}\left|\varkappa_{\dddot{A}_{\mathfrak{m}}} \in \mathbb{C},\right| \varkappa_{\dddot{A}_{\mathfrak{m}}} \mid \leq 1\right\}, \mathfrak{a}_{\ddot{A}_{\mathbb{C}}}: \dddot{\mathfrak{U}} \longrightarrow\left\{\varkappa_{\ddot{A}_{\mathfrak{a}}} \mid \varkappa_{\ddot{A}_{\mathfrak{a}}}\right.$ $\left.\in \mathbb{C},\left|\varkappa_{\ddot{A}_{\mathfrak{a}}}\right| \leq 1\right\}$, and $\mathfrak{n}_{\ddot{A}_{\mathbb{C}}}: \dddot{\mathfrak{U}} \longrightarrow\left\{\varkappa_{\ddot{A}_{\mathfrak{n}}}\left|\varkappa_{\ddot{A}_{\mathfrak{n}}} \in \mathbb{C},\right| \varkappa_{\ddot{A}_{\mathfrak{n}}} \mid \leq 1\right\}$ known as the degrees of membership, abstinence, and nonmembership, respectively, of $x \in \ddot{A}$ where $\varkappa_{\ddot{A}_{\mathfrak{m}}}(x)=\tau_{\ddot{A}_{\mathfrak{m}}}(x)$ $e^{\rho_{\ddot{A}_{\mathfrak{m}}}(x) 2 \pi i}$, $\varkappa_{\ddot{A}_{\mathfrak{a}}}(x)=\tau_{\ddot{A}_{\mathfrak{a}}}(x) e^{\rho_{\ddot{A}_{\mathfrak{a}}}(x) 2 \pi i}$, and $\varkappa_{\ddot{A}_{\mathfrak{n}}}(x)=\tau_{\ddot{A}_{\mathfrak{n}}}(x)$ $e^{\rho_{\ddot{A}_{\mathfrak{n}}}(x) 2 \pi i}$. A CNFS has the condition that $0 \leq\left|\mathbf{m}_{\ddot{A}_{\mathbb{C}}}(x)\right|+$ $\left|\mathfrak{a}_{\ddot{A}_{\mathbb{C}}}(x)\right|+\left|\mathfrak{n}_{\ddot{A}_{\mathbb{C}}}(x)\right| \leq 3 \quad$ or, equivalently, $\quad 0 \leq \tau_{\ddot{A}_{\mathfrak{m}}}(x)+\tau_{\ddot{A}_{\mathfrak{a}}}$ $(x)+\tau_{\ddot{A}_{\mathfrak{n}}}(x) \leq 3, \quad 0 \leq \rho_{\ddot{A}_{\mathfrak{m}}}(x)+\rho_{\ddot{A}_{\mathfrak{a}}}(x)+\rho_{\ddot{A}_{\mathfrak{n}}}(x) \leq 3 . \quad \tau_{\ddot{A}_{\mathfrak{m}}}(x)$, $\tau_{\ddot{A}_{\mathfrak{a}}}(x), \tau_{\ddot{A}_{\mathfrak{n}}}(x)$ are known as the amplitude terms, and $\rho_{\ddot{A}_{\mathfrak{m}}}(x)$, $\rho_{\ddot{A}_{\mathfrak{a}}}(x), \rho_{\ddot{A}_{\mathfrak{n}}}(x)$ are known as the phase terms.

\section{Main Results}

This section aims to define some new concepts in CNSs, like Cartesian product of CNSs and the CNRs. Moreover, types of CNRs are also introduced with examples. Furthermore, some interesting results and properties of these CNRs are obtained. 
Definition 11. The Cartesian product of two CNSs $\dddot{A}=\left\{x_{\mathbf{i}}, \mathfrak{m}_{\ddot{A}_{\mathbb{C}}}\left(x_{\mathrm{i}}\right), \mathfrak{a}_{\ddot{A}_{\mathbb{C}}}\left(x_{\mathrm{i}}\right), \mathfrak{n}_{\ddot{A}_{\mathbb{C}}}\left(x_{\mathrm{i}}\right) \mid x_{\mathrm{i}} \in \dddot{\mathfrak{U}}\right\} \quad$ and $\dddot{B}=\left\{x_{j}, \mathfrak{m}_{\dddot{B}_{\mathbb{C}}}\left(x_{j}\right), \quad \mathfrak{a}_{\ddot{B}_{\mathbb{C}}}\left(x_{j}\right), \mathfrak{n}_{\dddot{B}_{\mathbb{C}}}\left(x_{j}\right) \mid x_{j} \in \dddot{\mathfrak{U}}\right\}, \quad \mathrm{i}, j \in \mathbb{N}, \quad$ is given by

$$
\dddot{A} \times \dddot{B}=\left\{\left(x_{\mathrm{i}}, x_{j}\right), \mathfrak{m}_{(\dddot{A} \times \ddot{B})_{\mathbb{C}}}\left(x_{\mathbf{i}}, x_{j}\right), \mathfrak{a}_{(\dddot{A} \times \dddot{B})_{\mathbb{C}}}\left(x_{\mathbf{i}}, x_{j}\right), \mathfrak{n}_{(\dddot{A} \times \dddot{B})_{\mathbb{C}}}\left(x_{\mathbf{i}}, x_{j}\right) \mid x_{\mathbf{i}} \in \dddot{A}, x_{j} \in \dddot{B}\right\} .
$$

The functions $\mathfrak{m}_{(\dddot{A} \times \ddot{B})_{\mathbb{C}}}: \dddot{\mathfrak{U}} \longrightarrow\left\{\varkappa_{(\dddot{A} \times \ddot{B})_{\mathfrak{m}}} \mid \varkappa(\dddot{A} \times \bar{B})_{\mathfrak{m}} \in \mathbb{C}\right.$,

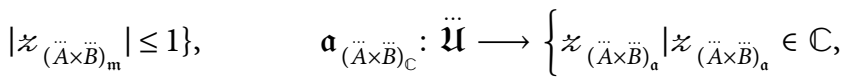

$\left.\left|\varkappa_{(\dddot{A} \times \ddot{B})_{\mathfrak{a}}}\right| \leq 1\right\}$, and $\mathfrak{n}_{(\dddot{A} \times \ddot{B})_{\mathbb{C}}}: \dddot{\mathfrak{U}} \longrightarrow\left\{\varkappa_{(\dddot{A} \times \ddot{B})_{\mathfrak{n}}} \mid \varkappa_{(\dddot{A} \times \ddot{B})_{\mathfrak{n}}} \in \mathbb{C}\right.$,

$\left.\left|\varkappa(\dddot{A} \times B)_{\mathfrak{n}}\right| \leq 1\right\}$ symbolize the degrees of membership, abstinence, andnonmembership of the Cartesian product $\dddot{A} \times \ddot{B}$. These functions are defined as

$$
\begin{aligned}
\mathfrak{m}_{(\ddot{A} \times \ddot{B})_{\mathbb{C}}}\left(x_{\mathbf{i}}, x_{j}\right) & =\min \left\{\mathfrak{m}_{\dddot{A}_{\mathbb{C}}}\left(x_{\mathbf{i}}\right), \mathfrak{m}_{\dddot{B}_{\mathbb{C}}}\left(x_{j}\right)\right\}, \\
\mathfrak{a}_{\left(\dddot{A} \times \dddot{B}_{\mathbb{C}}\right.}\left(x_{\mathbf{i}}, x_{j}\right) & =\min \left\{\mathfrak{a}_{\dddot{A}_{\mathbb{C}}}\left(x_{\mathbf{i}}\right), \mathfrak{a}_{\dddot{B}_{\mathbb{C}}}\left(x_{j}\right)\right\}, \\
\mathfrak{n}_{\left(\dddot{A} \times \dddot{B}_{\mathbb{C}}\right.}\left(x_{\mathbf{i}}, x_{j}\right) & =\max \left\{\mathfrak{n}_{\dddot{A}_{\mathbb{C}}}\left(x_{\mathbf{i}}\right), \mathfrak{n}_{\dddot{B}_{\mathbb{C}}}\left(x_{j}\right)\right\} .
\end{aligned}
$$

There is a condition that

$$
0 \leq\left|\mathbf{m}_{(\dddot{A} \times \dddot{B})_{\mathbb{C}}}\left(x_{\mathbf{i}}, x_{j}\right)\right|+\left|\mathfrak{a}_{(\dddot{A} \times \dddot{B})_{\mathbb{C}}}\left(x_{\mathbf{i}}, x_{j}\right)\right|+\left|\mathfrak{n}_{(\ddot{A} \times \ddot{B})_{\mathbb{C}}}\left(x_{\mathbf{i}}, x_{j}\right)\right| \leq 3 \text {. }
$$

Or equivalently

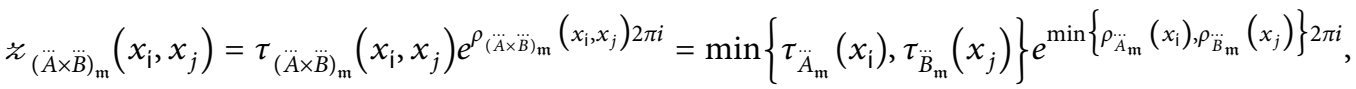

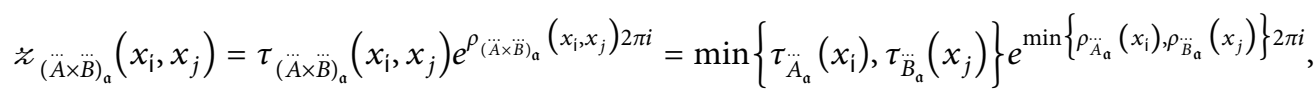

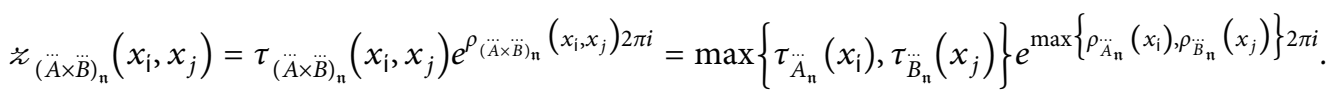

The conditions are $0 \leq \tau_{\dddot{A} \times \ddot{B}_{\mathfrak{m}}}\left(x_{\mathrm{i}}, x_{j}\right)+\tau_{\left(\dddot{A} \times \dddot{B}_{\mathfrak{a}}\right.}\left(x_{\mathrm{i}}, x_{j}\right)+$ $\tau_{(\dddot{A} \times \ddot{B})_{\mathfrak{n}}}\left(x_{\mathrm{i}}, x_{j}\right) \leq 3$ and $0 \leq \rho_{\ddot{A} \times \ddot{B}_{\mathfrak{m}}}\left(x_{\mathrm{i}}, x_{j}\right)+\rho_{\left(\ddot{A} \times \ddot{B}_{\mathfrak{a}}\right.}\left(x_{\mathrm{i}}, x_{j}\right)+$ $\rho_{(\ddot{A} \times B)_{\mathfrak{n}}}\left(x_{i}, x_{j}\right) \leq 3$, where

$$
\begin{aligned}
& \tau_{(\ddot{A} \times \ddot{B})_{\mathfrak{m}}}\left(x_{\mathbf{i}}, x_{j}\right), \tau_{(\dddot{A} \times \ddot{B})_{\mathfrak{a}}}\left(x_{\mathbf{i}}, x_{j}\right), \\
& \cdot \tau_{(\dddot{A} \times \ddot{B})_{\mathfrak{n}}}\left(x_{\mathbf{i}}, x_{j}\right), \rho_{(\dddot{A} \times \ddot{B})_{\mathfrak{m}}}\left(x_{\mathbf{i}}, x_{j}\right), \\
& \cdot \rho_{(\dddot{A} \times \dddot{B})_{\mathfrak{a}}}\left(x_{\mathbf{i}}, x_{j}\right), \quad \rho_{(\dddot{A} \times \ddot{B})_{\mathfrak{n}}}\left(x_{\mathbf{i}}, x_{j}\right) \in[0,1] .
\end{aligned}
$$

Definition 12. A complex neutrosophic relation (CNR) denoted by $\dddot{R}$ is any nonempty subset of $\dddot{A} \times \dddot{B}$, where $\dddot{A}$ and $\dddot{B}$ are CNSs.

Example 2. For a given CNS,

$$
\dddot{A}=\left\{\begin{array}{c}
\left(x, 0.8 e^{(0.6) 2 \pi i}, 1 e^{(0.1) 2 \pi i}, 1 e^{(0.5) 2 \pi i}\right),\left(y, 0 e^{(0.8) 2 \pi i}, 0.9 e^{(0.4) 2 \pi i}, 0.5 e^{(1) 2 \pi i}\right), \\
\left(\varkappa, 0.3 e^{(0.5) 2 \pi i}, 0.5 e^{(0.6) 2 \pi i}, 0.7 e^{(0.2) 2 \pi i}\right)
\end{array}\right\} .
$$


The Cartesian product of $\dddot{A}$ to itself is

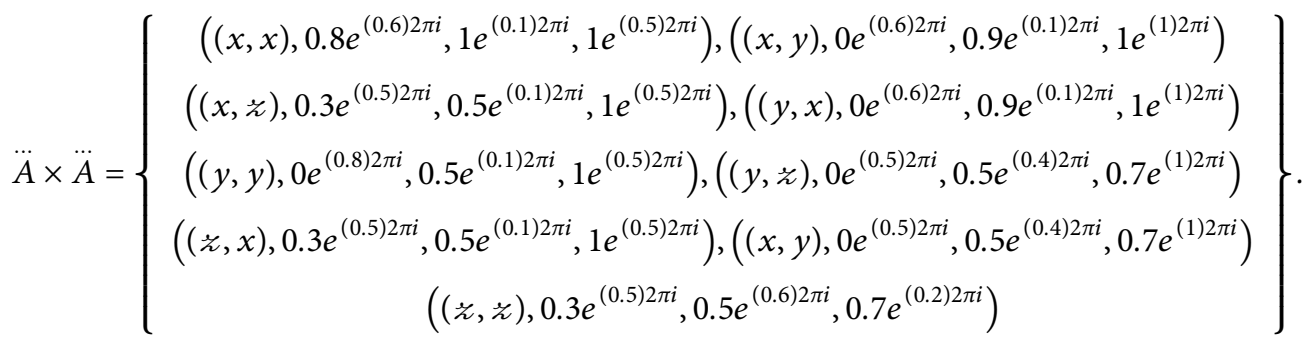

And the CNR $\dddot{\mathfrak{R}}$ is

$$
\dddot{\mathfrak{R}}=\left\{\begin{array}{c}
\left((x, \approx), 0.3 e^{(0.5) 2 \pi i}, 0.5 e^{(0.1) 2 \pi i}, 1 e^{(0.5) 2 \pi i}\right),\left((y, y), 0 e^{(0.8) 2 \pi i}, 0.9 e^{(0.4) 2 \pi i}, 0.5 e^{(1) 2 \pi i}\right), \\
\left((\varkappa, y), 0 e^{(0.5) 2 \pi i}, 0.5 e^{(0.4) 2 \pi i}, 0.7 e^{(1) 2 \pi i}\right)
\end{array}\right\} .
$$

Definition 13. The inverse CNR $\dddot{R}^{-1}$ of a CNFR

$$
\dddot{\mathfrak{R}}=\left\{\left(x_{\mathrm{i}}, x_{j}\right), \mathfrak{m}_{\mathfrak{R}_{\mathbb{C}}}\left(x_{\mathrm{i}}, x_{j}\right), \mathfrak{a}_{\mathfrak{R}_{\mathbb{C}}}\left(x_{i}, x_{j}\right), \mathfrak{n}_{\mathfrak{R}_{\mathbb{C}}}\left(x_{\mathrm{i}}, x_{j}\right) \mid\left(x_{\mathrm{i}}, x_{j}\right) \in \dddot{\mathfrak{R}}\right\},
$$

is defined as

$$
\dddot{\mathfrak{R}}^{-1}=\left\{\left(x_{\mathrm{i}}, x_{j}\right), \mathfrak{m}_{\dddot{\mathfrak{R}}_{\mathbb{C}}}\left(x_{\mathrm{i}}, x_{j}\right), \mathfrak{a}_{\mathfrak{R}_{\mathbb{C}}}\left(x_{\mathrm{i}}, x_{j}\right), \mathfrak{n}_{\mathfrak{R}_{\mathbb{C}}}\left(x_{\mathrm{i}}, x_{j}\right) \mid\left(x_{\mathrm{i}}, x_{j}\right) \in \dddot{\mathfrak{R}}\right\} .
$$

Example 3. For a CNR $\dddot{R}$ from (12),

$$
\dddot{\mathfrak{R}}=\left\{\left((y, x), 0 e^{(0.6) 2 \pi i}, 0.9 e^{(0.1) 2 \pi i}, 1 e^{(1) 2 \pi i}\right),\left((y, \approx), 0 e^{(0.5) 2 \pi i}, 0.5 e^{(0.4) 2 \pi i}, 0.7 e^{(1) 2 \pi i}\right)\right\}
$$

The inverse CNR $\dddot{\mathfrak{R}}^{-1}$ is given by

$$
\dddot{\mathfrak{R}}^{-1}=\left\{\left((y, x), 0 e^{(0.6) 2 \pi i}, 0.9 e^{(0.1) 2 \pi i}, 1 e^{(1) 2 \pi i}\right),\left((y, \varkappa), 0 e^{(0.5) 2 \pi i}, 0.5 e^{(0.4) 2 \pi i}, 0.7 e^{(1) 2 \pi i}\right)\right\} .
$$

Definition 14. A CNR $\dddot{R}_{1}$ is said to be a CN reflexive relation if

$$
\forall\left(x, \mathfrak{m}_{\ddot{A}_{\mathbb{C}}}(x), \mathfrak{a}_{\ddot{A}_{\mathbb{C}}}(x), \mathfrak{n}_{\ddot{A}_{\mathscr{C}}}(x)\right) \in \dddot{A} \Rightarrow\left((x, x), \mathfrak{m}_{\dddot{A}_{\mathbb{C}}}(x, x), \mathfrak{a}_{\ddot{A}_{\mathbb{C}}}(x, x), \mathfrak{n}_{\dddot{A}_{\mathbb{C}}}(x, x)\right) \in \dddot{\mathfrak{R}}_{1} .
$$


While on the other hand a CN irreflexive relation $\dddot{\mathfrak{R}}_{2}$ implies

$$
\begin{aligned}
& \left((x, x), \mathfrak{m}_{\dddot{A}_{\mathbb{C}}}(x, x), \mathfrak{a}_{\dddot{A}_{\mathbb{C}}}(x, x), \mathfrak{n}_{\dddot{A}_{\mathbb{C}}}(x, x)\right) \notin \dddot{\mathfrak{R}}_{2}, \\
& \cdot \forall\left(x, \mathfrak{m}_{\dddot{A}_{\mathbb{C}}}(x), \mathfrak{a}_{\dddot{A}_{\mathbb{C}}}(x), \mathfrak{n}_{\ddot{A}_{C}}(x)\right) \in \dddot{A} .
\end{aligned}
$$

$$
\begin{aligned}
& \dddot{\mathfrak{R}}_{1}=\left\{\begin{array}{c}
\left((x, x), 0.8 e^{(0.6) 2 \pi i}, 1 e^{(0.1) 2 \pi i}, 1 e^{(0.5) 2 \pi i}\right),\left((y, y), 0 e^{(0.8) 2 \pi i}, 0.9 e^{(0.4) 2 \pi i}, 0.5 e^{(1) 2 \pi i}\right), \\
\left((\varkappa, \varkappa), 0.3 e^{(0.5) 2 \pi i}, 0.5 e^{(0.6) 2 \pi i}, 0.7 e^{(0.2) 2 \pi i}\right)
\end{array}\right\}, \\
& \dddot{\mathfrak{R}}_{2}=\left\{\left((x, y), 0 e^{(0.6) 2 \pi i}, 0.9 e^{(0.1) 2 \pi i}, 1 e^{(1) 2 \pi i}\right),\left((\varkappa, x), 0.3 e^{(0.5) 2 \pi i}, 0.5 e^{(0.1) 2 \pi i}, 1 e^{(0.5) 2 \pi i}\right)\right\} .
\end{aligned}
$$

Definition 15. A CNR $\dddot{R}$ is said to be CN symmetric relation if

$$
\begin{aligned}
& \forall\left((x, y), \mathfrak{m}_{\dddot{R}_{\mathbb{C}}}(x, y), \mathfrak{a}_{\mathfrak{R}_{\mathbb{C}}}(x, y), \mathfrak{n}_{\dddot{R}_{\mathbb{C}}}(x, y)\right) \in \dddot{\mathfrak{R}} \\
& \quad \Rightarrow\left((y, x), \mathfrak{m}_{\dddot{\mathfrak{R}}_{\mathbb{C}}}(y, x), \mathfrak{a}_{\dddot{R}_{\mathbb{C}}}(y, x), \mathfrak{n}_{\dddot{\mathfrak{R}}_{\mathbb{C}}}(y, x)\right) \in \dddot{\mathfrak{R}} .
\end{aligned}
$$

Example 4. Using (12), the CN reflexive relation $\dddot{\mathfrak{R}}_{1}$ and the $\mathrm{CN}$ irreflexive relation $\dddot{\mathfrak{R}}_{2}$ are
A CNR $\dddot{R}_{1}$ is said to be CN asymmetric relation if

$$
\begin{aligned}
& \forall\left((x, y), \mathfrak{m}_{\dddot{R}_{\mathbb{C}}}(x, y), \mathfrak{a}_{\mathfrak{R}_{\mathbb{C}}}(x, y), \mathfrak{n}_{\dddot{R}_{\mathbb{C}}}(x, y)\right) \in \dddot{\mathfrak{R}}_{1} \\
& \quad \Rightarrow\left((y, x), \mathfrak{m}_{\dddot{R}_{\mathbb{C}}}(y, x), \mathfrak{a}_{\dddot{\mathfrak{R}}_{\mathbb{C}}}(y, x), \mathfrak{n}_{\dddot{\mathfrak{R}}_{\mathbb{C}}}(y, x)\right) \notin \dddot{\mathfrak{R}}_{1} .
\end{aligned}
$$

If

$$
\begin{aligned}
& \left((x, y), \mathfrak{m}_{\dddot{R}_{\mathbb{C}}}(x, y), \mathfrak{a}_{\mathfrak{R}_{\mathbb{C}}}(x, y), \mathfrak{n}_{\dddot{\mathfrak{R}}_{\mathbb{C}}}(x, y)\right) \in \dddot{\mathfrak{R}}_{2}, \\
& \left((y, x), \mathfrak{m}_{\mathfrak{R}_{\mathbb{C}}}(y, x), \mathfrak{a}_{\mathfrak{R}_{\mathbb{C}}}(y, x), \mathfrak{n}_{\mathfrak{R}_{\mathbb{C}}}(y, x)\right) \in \dddot{\mathfrak{R}}_{2}, \\
& \Rightarrow\left((x, y), \mathfrak{m}_{\mathfrak{R}_{C}}(x, y), \mathfrak{a}_{\dddot{R}_{C}}(x, y), \mathfrak{n}_{\mathfrak{R}_{\mathbb{C}}}(x, y)\right)=\left((y, x), \mathfrak{m}_{\mathfrak{R}_{\mathbb{C}}}(y, x), \mathfrak{a}_{\mathfrak{R}_{\mathbb{C}}}(y, x), \mathfrak{n}_{\mathfrak{R}_{C}}(y, x)\right) .
\end{aligned}
$$

then $\dddot{\mathfrak{R}}_{2}$ is a $\mathrm{CN}$ antisymmetric relation.

Example 5. Using (12), the $\mathrm{CN}$ symmetric relation $\dddot{\mathfrak{R}}$, the $\mathrm{CN}$ asymmetric relation $\dddot{R}_{1}$, and the $\mathrm{CN}$ antisymmetric relation $\dddot{\mathfrak{R}}_{2}$ are

$$
\begin{aligned}
& \dddot{\mathfrak{R}}=\left\{\begin{array}{c}
\left((x, y), 0 e^{(0.6) 2 \pi i}, 0.9 e^{(0.1) 2 \pi i}, 1 e^{(1) 2 \pi i}\right),\left((x, \varkappa), 0.3 e^{(0.5) 2 \pi i}, 0.5 e^{(0.1) 2 \pi i}, 1 e^{(0.5) 2 \pi i}\right), \\
\left((y, \approx), 0 e^{(0.6) 2 \pi i}, 0.9 e^{(0.1) 2 \pi i}, 1 e^{(1) 2 \pi i}\right),\left((\varkappa, x), 0.3 e^{(0.5) 2 \pi i}, 0.5 e^{(0.1) 2 \pi i}, 1 e^{(0.5) 2 \pi i}\right)
\end{array}\right\},
\end{aligned}
$$

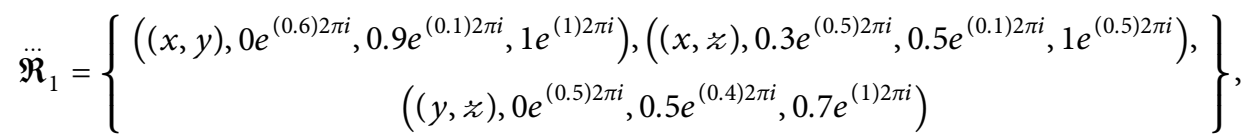

$$
\begin{aligned}
& \dddot{\Re}_{2}=\left\{\left((x, x), 0.8 e^{(0.6) 2 \pi i}, 1 e^{(0.1) 2 \pi i}, 1 e^{(0.5) 2 \pi i}\right),\left((y, y), 0 e^{(0.8) 2 \pi i}, 0.9 e^{(0.4) 2 \pi i}, 0.5 e^{(1) 2 \pi i}\right)\right\} .
\end{aligned}
$$

Theorem 1. A CNR $\dddot{\mathfrak{R}}$ is a symmetric relation iff $\dddot{\mathfrak{R}}=\dddot{\mathfrak{R}}^{-1}$.

Proof. Suppose that $\dddot{R}=\dddot{\mathfrak{R}}^{-1}$, then

$$
\begin{aligned}
& \left((x, y), \mathfrak{m}_{\dddot{\mathfrak{R}}_{\mathbb{C}}}(x, y), \mathfrak{a}_{\mathfrak{R}_{\mathbb{C}}}(x, y), \mathfrak{n}_{\dddot{R}_{\mathbb{C}}}(x, y)\right) \in \dddot{\mathfrak{R}} \\
& \quad \Rightarrow\left((y, x), \mathfrak{m}_{\dddot{R}_{\mathbb{C}}}(y, x), \mathfrak{a}_{\dddot{R}_{\mathbb{C}}}(y, x), \mathfrak{n}_{\dddot{R}_{\mathbb{C}}}(y, x)\right) \in \dddot{\mathfrak{R}}^{-1} .
\end{aligned}
$$




$$
\begin{aligned}
& \text { But } \dddot{\mathfrak{R}}=\dddot{\mathfrak{R}}^{-1} . \\
& \Rightarrow\left((y, x), \mathfrak{m}_{\dddot{\mathfrak{R}}_{\mathbb{C}}}(y, x), \mathfrak{a}_{\dddot{\mathfrak{R}}_{\mathbb{C}}}(y, x), \mathfrak{n}_{\dddot{\mathfrak{R}}_{\mathbb{C}}}(y, x)\right) \in \dddot{\mathfrak{R}} .
\end{aligned}
$$

Hence, $\dddot{R}$ is a $\mathrm{CN}$ symmetric relation.

Conversely, suppose that $\mathfrak{R}$ is a CN symmetric relation, then for

$$
\begin{aligned}
& \left((x, y), \mathfrak{m}_{\dddot{R}_{\mathbb{C}}}(x, y), \mathfrak{a}_{\dddot{R}_{\mathbb{C}}}(x, y), \mathfrak{n}_{\mathfrak{\Re}_{\mathbb{C}}}(x, y)\right) \in \dddot{\mathfrak{R}} \\
& \Rightarrow\left((y, x), \mathfrak{m}_{\dddot{\Re}_{\mathbb{C}}}(y, x), \mathfrak{a}_{\dddot{\mathfrak{R}}_{\mathbb{C}}}(y, x), \mathfrak{n}_{\dddot{\mathfrak{R}}_{\mathbb{C}}}(y, x)\right) \in \mathfrak{\Re} .
\end{aligned}
$$

But

$$
\begin{aligned}
\left((x, y), \mathfrak{m}_{\mathfrak{R}_{\mathbb{C}}}(x, y), \mathfrak{a}_{\dddot{R}_{\mathbb{C}}}(x, y), \mathfrak{n}_{\mathfrak{R}_{\mathbb{C}}}(x, y)\right) \in \dddot{\mathfrak{R}} & \Rightarrow\left((y, x), \mathfrak{m}_{\mathfrak{R}_{\mathbb{C}}}(y, x), \mathfrak{a}_{\mathfrak{\Re}_{\mathbb{C}}}(y, x), \mathfrak{n}_{\mathfrak{R}_{\mathbb{C}}}(y, x)\right) \in \dddot{\mathfrak{R}}^{-1} \\
& \Rightarrow \dddot{\mathfrak{R}}=\dddot{\mathfrak{R}}^{-1}
\end{aligned}
$$

Theorem 2. For CN symmetric relations $\dddot{\mathfrak{R}}_{1}$ and $\dddot{\mathfrak{R}}_{2}$, the intersection $\dddot{\mathfrak{R}}_{1} \cap \dddot{\mathfrak{R}}_{2}$ is also a CN symmetric relation.

Proof. Suppose that $\dddot{R}_{1}$ and $\dddot{R}_{2}$ are two CN symmetric relations on a CNS $\dddot{A}$. Then according to the definition of $\mathrm{CNR}, \quad \dddot{R}_{1} \subseteq \dddot{A} \times \dddot{A}$ and $\dddot{\mathfrak{R}}_{2} \subseteq \dddot{A} \times \dddot{A} \Rightarrow \dddot{\mathfrak{R}}_{1} \cap \dddot{\mathfrak{R}}_{2} \subseteq \dddot{A} \times \dddot{A} \Rightarrow$ $\dddot{R}_{1} \cap \dddot{\Re}_{2}$ is CNR on $\dddot{A}$.

Now, suppose that

$$
\begin{gathered}
\left((x, y), \mathfrak{m}_{\mathfrak{R}_{C}}(x, y), \mathfrak{a}_{\mathfrak{R}_{C}}(x, y), \mathfrak{n}_{\dddot{R}_{C}}(x, y)\right) \in \dddot{R}_{1} \cap \dddot{\Re}_{2} \\
\Rightarrow\left((x, y), \mathfrak{m}_{\dddot{R}_{C}}(x, y), \mathfrak{a}_{\mathfrak{\Re}_{C}}(x, y), \mathfrak{n}_{\mathfrak{R}_{C}}(x, y)\right) \in \dddot{\Re}_{1} \\
\cdot\left((x, y), \mathfrak{m}_{\mathfrak{R}_{C}}(x, y), \mathfrak{a}_{\mathfrak{R}_{C}}(x, y), \mathfrak{n}_{\mathfrak{R}_{C}}(x, y)\right) \in \dddot{\mathfrak{R}}_{2}
\end{gathered}
$$

Since $\dddot{\mathfrak{R}}_{1}$ and $\dddot{\mathfrak{R}}_{2}$ are CN symmetric relations,

$$
\begin{aligned}
& \left((y, x), \mathfrak{m}_{\dddot{R}_{\mathbb{C}}}(y, x), \mathfrak{a}_{\mathfrak{R}_{\mathbb{C}}}(y, x), \mathfrak{n}_{\mathfrak{R}_{\mathbb{C}}}(y, x)\right) \in \dddot{\mathfrak{R}}_{1}, \\
& \left((y, x), \mathfrak{m}_{\dddot{R}_{\mathbb{C}}}(y, x), \mathfrak{a}_{\mathfrak{\Re}_{\mathbb{C}}}(y, x), \mathfrak{n}_{\dddot{\Re}_{\mathbb{C}}}(y, x)\right) \in \dddot{\mathfrak{R}}_{2}, \\
& \quad \Rightarrow\left((y, x), \mathfrak{m}_{\dddot{\mathfrak{R}}_{\mathbb{C}}}(y, x), \mathfrak{a}_{\mathfrak{\Re}_{\mathbb{C}}}(y, x), \mathfrak{n}_{\mathfrak{\Re}_{\mathbb{C}}}(y, x)\right) \in \dddot{\mathfrak{R}}_{1} \cap \dddot{\mathfrak{R}}_{2} .
\end{aligned}
$$

Definition 16. A CNR $\dddot{R}$ is said to be $\mathrm{CN}$ transitive relation if

$$
\begin{aligned}
& \left((x, y), \mathfrak{m}_{\dddot{R}_{\mathbb{C}}}(x, y), \mathfrak{a}_{\dddot{R}_{\mathbb{C}}}(x, y), \mathfrak{n}_{\dddot{R}_{\mathbb{C}}}(x, y)\right) \in \dddot{\mathfrak{R}}, \\
& \left((y, \varkappa), \mathfrak{m}_{\mathfrak{R}_{\mathbb{C}}}(y, \varkappa), \mathfrak{a}_{\mathfrak{R}_{\mathbb{C}}}(y, \varkappa), \mathfrak{n}_{\dddot{R}_{\mathbb{C}}}(y, \varkappa)\right) \in \dddot{\mathfrak{R}}, \\
& \Rightarrow\left((x, \varkappa), \mathfrak{m}_{\mathfrak{R}_{\mathbb{C}}}(x, \varkappa), \mathfrak{a}_{\mathfrak{R}_{\mathbb{C}}}(x, \hbar), \mathfrak{n}_{\mathfrak{R}_{\mathbb{C}}}(x, \varkappa)\right) \in \dddot{\mathfrak{R}} .
\end{aligned}
$$

Example 6. A CN transitive relation $\dddot{R}$ on (12) is

$$
\dddot{R}=\left\{\begin{array}{c}
\left((x, y), 0 e^{(0.6) 2 \pi i}, 0.9 e^{(0.1) 2 \pi i}, 1 e^{(1) 2 \pi i}\right),\left((x, \approx), 0.3 e^{(0.5) 2 \pi i}, 0.5 e^{(0.1) 2 \pi i}, 1 e^{(0.5) 2 \pi i}\right), \\
\left((\varkappa, y), 0 e^{(0.5) 2 \pi i}, 0.5 e^{(0.4) 2 \pi i}, 0.7 e^{(1) 2 \pi i}\right)
\end{array}\right\} .
$$

Definition 17. A CN composite relation $\dddot{R}_{1} \circ \dddot{R}_{2}$ combines the CNRs $\dddot{\mathfrak{R}}_{1}$ and $\dddot{\mathfrak{R}}_{2}$ such that for $\left((x, y), \mathfrak{m}_{\mathfrak{R}_{\mathbb{C}}}(x, y)\right.$,
$\left.\mathfrak{a}_{\mathfrak{R}_{C}}(x, y), \mathfrak{n}_{\mathfrak{R}_{\mathbb{C}}}(x, y)\right) \in \dddot{\mathfrak{R}}_{1} \quad$ and $\quad\left((y, z), \mathfrak{m}_{\dddot{\mathfrak{R}}_{\mathrm{C}}}(y, z), \mathfrak{a}_{\mathfrak{\Re}_{\mathrm{C}}}\right.$ $(y$, $), \mathfrak{n}_{\dddot{R}_{C}}(y$, $\left.)\right) \in \dddot{\mathfrak{R}}_{2}$, 


$$
\left((x, \approx), \mathfrak{m}_{\mathfrak{\Re}_{C}}(x, \approx), \mathfrak{a}_{\mathfrak{R}_{C}}(x, \approx), \mathfrak{n}_{\mathfrak{R}_{C}}(x, \approx)\right) \in \dddot{\mathfrak{R}}_{1} \circ \dddot{\mathfrak{R}}_{2} . \quad \text { Example 7. For CNRs } \dddot{\mathfrak{R}}_{1} \text { and } \dddot{\mathfrak{R}}_{2} \text {, }
$$

$$
\begin{aligned}
& \dddot{\mathfrak{R}}_{1}=\left\{\left((x, y), 0 e^{(0.6) 2 \pi i}, 0.9 e^{(0.1) 2 \pi i}, 1 e^{(1) 2 \pi i}\right),\left((x, \approx), 0.3 e^{(0.5) 2 \pi i}, 0.5 e^{(0.1) 2 \pi i}, 1 e^{(0.5) 2 \pi i}\right)\right\}, \\
& \dddot{R}_{2}=\left\{\begin{array}{c}
\left((y, x), 0 e^{(0.6) 2 \pi i}, 0.9 e^{(0.1) 2 \pi i}, 1 e^{(1) 2 \pi i}\right),\left((y, \approx), 0 e^{(0.5) 2 \pi i}, 0.5 e^{(0.4) 2 \pi i}, 0.7 e^{(1) 2 \pi i}\right), \\
\left((\approx, y), 0 e^{(0.5) 2 \pi i}, 0.5 e^{(0.4) 2 \pi i}, 0.7 e^{(1) 2 \pi i}\right)
\end{array}\right\} .
\end{aligned}
$$

The $\mathrm{CN}$ composite relation is

$$
\dddot{\Re}_{1} \circ \dddot{R}_{2}=\left\{\begin{array}{c}
\left((x, x), 0.8 e^{(0.6) 2 \pi i}, 1 e^{(0.1) 2 \pi i}, 1 e^{(0.5) 2 \pi i}\right),\left((x, \approx), 0.3 e^{(0.5) 2 \pi i}, 0.5 e^{(0.1) 2 \pi i}, 1 e^{(0.5) 2 \pi i}\right), \\
\left((x, y), 0 e^{(0.6) 2 \pi i}, 0.9 e^{(0.1) 2 \pi i}, 1 e^{(1) 2 \pi i}\right)
\end{array}\right\} .
$$

Theorem 3. A CNR $\dddot{R}$ is a transitive relation iff $\dddot{R} \circ \dddot{\Re} \subseteq \dddot{R}$.

Proof. Suppose that $\dddot{R}$ is a CN transitive relation, then for $\left((x, y), \mathfrak{m}_{\mathfrak{R}_{C}}(x, y), \mathfrak{a}_{\mathfrak{R}_{\mathbb{C}}}(x, y), \mathfrak{n}_{\mathfrak{R}_{C}}(x, y)\right) \in \dddot{\mathfrak{R}}$ and $((y, \approx)$, $\left.\mathfrak{m}_{\mathfrak{R}_{\mathbb{C}}}(y, \approx), \mathfrak{a}_{\mathfrak{R}_{C}}(y, \approx), \mathfrak{n}_{\mathfrak{R}_{\mathbb{C}}}(y, \approx)\right) \in \dddot{R}$,

$$
\Rightarrow\left((x, \approx), \mathfrak{m}_{\mathfrak{R}_{\mathbb{C}}}(x, \approx), \mathfrak{a}_{\mathfrak{R}_{\mathbb{C}}}(x, \approx), \mathfrak{n}_{\mathfrak{R}_{\mathbb{C}}}(x, \approx)\right) \in \dddot{\mathfrak{R}} .
$$

But $\left((x, \approx), \mathfrak{m}_{\mathfrak{R}_{C}}(x, \approx), \mathfrak{a}_{\mathfrak{R}_{C}}(x, \approx), \mathfrak{n}_{\mathfrak{R}_{C}}(x, \approx)\right) \in \dddot{R} \circ \dddot{R}$. Hence $\dddot{R} \circ \dddot{R} \subseteq \dddot{R}$.

Conversely, suppose that $\dddot{R} \circ \dddot{R} \subseteq \dddot{R}$, then for $((x, y)$, $\left.\mathfrak{m}_{\mathfrak{R}_{\mathbb{C}}}(x, y), \mathfrak{a}_{\mathfrak{R}_{\mathbb{C}}}(x, y), \mathfrak{n}_{\mathfrak{R}_{\mathbb{C}}}(x, y)\right) \in \dddot{\mathfrak{R}} \quad$ and $\quad\left((y, z), \mathfrak{m}_{\mathfrak{R}_{\mathbb{C}}}\right.$ $\left.(y, \approx), \mathfrak{a}_{\mathfrak{R}_{\mathbb{C}}}(y, \approx), \mathfrak{n}_{\mathfrak{R}_{\mathbb{C}}}(y, \approx)\right) \in \dddot{\mathfrak{R}}$,

$$
\Rightarrow\left((x, \approx), \mathfrak{m}_{\dddot{R}_{C}}(x, \approx), \mathfrak{a}_{\mathfrak{\Re}_{C}}(x, \approx), \mathfrak{n}_{\mathfrak{R}_{C}}(x, \approx)\right) \in \dddot{\mathfrak{R}} \circ \dddot{\Re} .
$$

But $\quad \dddot{R} \circ \dddot{R} \subseteq \dddot{R} \Rightarrow\left((x, \approx), \mathfrak{m}_{\mathfrak{R}_{\mathbb{C}}}(x, \approx), \mathfrak{a}_{\mathfrak{R}_{C}}(x, \approx), \mathfrak{n}_{\mathfrak{R}_{\mathbb{C}}}(x\right.$, そ) $) \in \dddot{R}$

Thus, $\dddot{R}$ is a $\mathrm{CN}$ transitive relation.

Definition 18. If a CNR $\dddot{R}_{1}$ satisfies the conditions of CN reflexive relation, $\mathrm{CN}$ symmetric relation, and $\mathrm{CN}$ transitive relation, then $\dddot{R}_{1}$ is called a $\mathrm{CN}$ equivalence relation.

While a CNR $\dddot{R}_{2}$ satisfying the conditions of CN reflexive relation, $\mathrm{CN}$ antisymmetric relation and $\mathrm{CN}$ transitive relation are called a $\mathrm{CN}$-order relation.

Example 8. A CN equivalence relation $\dddot{R}$ on (12) is

$$
\dddot{R}=\left\{\begin{array}{c}
\left((x, x), 0.8 e^{(0.6) 2 \pi i}, 1 e^{(0.1) 2 \pi i}, 1 e^{(0.5) 2 \pi i}\right),\left((x, \approx), 0.3 e^{(0.5) 2 \pi i}, 0.5 e^{(0.1) 2 \pi i}, 1 e^{(0.5) 2 \pi i}\right), \\
\left((y, y), 0 e^{(0.8) 2 \pi i}, 0.9 e^{(0.4) 2 \pi i}, 0.5 e^{(1) 2 \pi i}\right),\left((\varkappa, x), 0.3 e^{(0.5) 2 \pi i}, 0.5 e^{(0.1) 2 \pi i}, 1 e^{(0.5) 2 \pi i}\right), \\
\left((\varkappa, \approx), 0.3 e^{(0.5) 2 \pi i}, 0.5 e^{(0.6) 2 \pi i}, 0.7 e^{(0.2) 2 \pi i}\right)
\end{array}\right\} .
$$

And a CN-order relation $\dddot{R}$ on (12) is

$$
\dddot{\Re}=\left\{\begin{array}{c}
\left((x, x), 0.8 e^{(0.6) 2 \pi i}, 1 e^{(0.1) 2 \pi i}, 1 e^{(0.5) 2 \pi i}\right),\left((x, y), 0 e^{(0.6) 2 \pi i}, 0.9 e^{(0.1) 2 \pi i}, 1 e^{(1) 2 \pi i}\right), \\
\left((x, \approx), 0.3 e^{(0.5) 2 \pi i}, 0.5 e^{(0.1) 2 \pi i}, 1 e^{(0.5) 2 \pi i}\right),\left((y, y), 0 e^{(0.8) 2 \pi i}, 0.9 e^{(0.4) 2 \pi i}, 0.5 e^{(1) 2 \pi i}\right), \\
\left((\varkappa, y), 0 e^{(0.5) 2 \pi i}, 0.5 e^{(0.4) 2 \pi i}, 0.7 e^{(1) 2 \pi i}\right),\left((\text { ๘ }), 0.3 e^{(0.5) 2 \pi i}, 0.5 e^{(0.6) 2 \pi i}, 0.7 e^{(0.2) 2 \pi i}\right)
\end{array}\right\}
$$


Theorem 4. A CN equivalence relation implies that $\dddot{R}=\dddot{R} \circ \dddot{R}$

Proof. Since a CN equivalence relation is $\dddot{R}$ also a CN transitive relation, by Theorem 3 ,

$$
\dddot{R} \circ \dddot{R} \subseteq \dddot{R} \text {. }
$$

Now suppose that

$$
\left((x, y), \mathfrak{m}_{\mathfrak{R}_{\mathbb{C}}}(x, y), \mathfrak{a}_{\mathfrak{R}_{\mathbb{C}}}(x, y), \mathfrak{n}_{\mathfrak{R}_{\mathbb{C}}}(x, y)\right) \in \dddot{R} .
$$

As $\dddot{R}$ is an equivalence relation and possesses the properties of $\mathrm{CN}$ symmetric relation and $\mathrm{CN}$ transitive relation,

$$
\Rightarrow\left((y, x), \mathfrak{m}_{\mathfrak{R}_{\mathbb{C}}}(y, x), \mathfrak{a}_{\mathfrak{R}_{\mathbb{C}}}(y, x), \mathfrak{n}_{\mathfrak{R}_{\mathbb{C}}}(y, x)\right) \in \dddot{\mathfrak{R}},
$$

(41) and (42) imply that

$$
\left((x, x), \mathfrak{m}_{\ddot{A}_{C}}(x, x), \mathfrak{a}_{\ddot{A}_{C}}(x, x), \mathfrak{n}_{\ddot{A}_{C}}(x, x)\right) \in \dddot{\Re} .
$$

But according to $\mathrm{CN}$ composite relation,

$$
\begin{array}{r}
\left((x, x), \mathfrak{m}_{\ddot{A}_{C}}(x, x), \mathfrak{a}_{\ddot{A}_{C}}(x, x), \mathfrak{n}_{\ddot{A}_{C}}(x, x)\right) \\
\in \dddot{\mathfrak{R}} \circ \dddot{\mathfrak{R}} \\
\Rightarrow \dddot{\mathfrak{R}} \subseteq \dddot{\mathfrak{R}} \circ \dddot{\mathfrak{R}},
\end{array}
$$

(40) and (41) imply that $\dddot{R}=\dddot{R} \circ \dddot{R}$.
Theorem 5. The inverse $C N R \dddot{\Re}^{-1}$ of a $C N$-order relation $\dddot{R}$ is also a $\mathrm{CN}$-order relation.

Proof. The inverse CNR $\dddot{R}^{-1}$ of a CN order relation $\dddot{R}$ is also a $\mathrm{CN}$ order relation if the three properties for a $\mathrm{CN}$ order relation hold:

(1) $\forall x \in \dddot{A}, \quad\left((x, x), \mathfrak{m}_{\ddot{A}_{C}}(x, x), \mathfrak{a}_{\ddot{A}_{C}}(x, x), \mathfrak{n}_{\ddot{A}_{C}}(x, x)\right) \epsilon$ $\dddot{R} \neq$ because $\dddot{R}$ is also a $C N$ reflexive relation:

$$
\Rightarrow\left((x, x), \mathfrak{m}_{\dddot{A}_{\mathbb{C}}}(x, x), \mathfrak{a}_{\ddot{A}_{\mathbb{C}}}(x, x), \mathfrak{n}_{\dddot{A}_{\mathbb{C}}}(x, x)\right) \in \dddot{\mathfrak{R}}^{-1} .
$$

Hence, $\dddot{R}^{-1}$ is a CN reflexive relation.

(2) Suppose

$$
\begin{aligned}
& \left((x, y), \mathfrak{m}_{\mathfrak{R}_{\mathrm{C}}}(x, y), \mathfrak{a}_{\mathfrak{R}_{\mathrm{C}}}(x, y), \mathfrak{n}_{\mathfrak{\Re}_{\mathrm{C}}}(x, y)\right) \in \dddot{\mathfrak{R}}, \\
& \left((y, x), \mathfrak{m}_{\mathfrak{R}_{\mathrm{C}}}(y, x), \mathfrak{a}_{\mathfrak{R}_{\mathrm{C}}}(y, x), \mathfrak{n}_{\mathfrak{R}_{\mathrm{C}}}(y, x)\right) \in \dddot{\mathfrak{R}}, \\
& \Rightarrow\left((y, x), \mathfrak{m}_{\mathfrak{R}_{\mathrm{C}}}(y, x), \mathfrak{a}_{\mathfrak{R}_{\mathbb{C}}}(y, x), \mathfrak{n}_{\mathfrak{R}_{\mathbb{C}}}(y, x)\right) \in \dddot{\mathfrak{R}}^{-1}, \\
& \left((x, y), \mathfrak{m}_{\mathfrak{R}_{\mathbb{C}}}(x, y), \mathfrak{a}_{\mathfrak{R}_{\mathbb{C}}}(x, y), \mathfrak{n}_{\mathfrak{R}_{\mathbb{C}}}(x, y)\right) \in \dddot{\mathfrak{R}}^{-1} .
\end{aligned}
$$

But $\dddot{R}$ is also a $\mathrm{CN}$ antisymmetric relation. So

$$
\left((x, y), \mathfrak{m}_{\mathfrak{R}_{C}}(x, y), \mathfrak{a}_{\mathfrak{R}_{C}}(x, y), \mathfrak{n}_{\mathfrak{R}_{C}}(x, y)\right)=\left((y, x), \mathfrak{m}_{\mathfrak{R}_{C}}(y, x), \mathfrak{a}_{\mathfrak{R}_{C}}(y, x), \mathfrak{n}_{\mathfrak{R}_{C}}(y, x)\right) .
$$

Hence, $\dddot{\mathfrak{R}}^{-1}$ is a $\mathrm{CN}$ antisymmetric relation.

(3) Suppose

$$
\begin{aligned}
& \left((x, y), \mathfrak{m}_{\mathfrak{R}_{\mathbb{C}}}(x, y), \mathfrak{a}_{\dddot{\mathfrak{R}}_{\mathrm{C}}}(x, y), \mathfrak{n}_{\mathfrak{R}_{\mathbb{C}}}(x, y)\right) \in \dddot{\mathfrak{R}}
\end{aligned}
$$

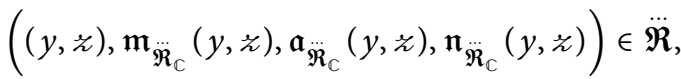

$$
\begin{aligned}
& \Rightarrow\left((y, x), \mathfrak{m}_{\mathfrak{R}_{\mathbb{C}}}(y, x), \mathfrak{a}_{\mathfrak{R}_{\mathbb{C}}}(y, x), \mathfrak{n}_{\mathfrak{R}_{\mathbb{C}}}(y, x)\right) \in \dddot{\mathfrak{R}}^{-1},
\end{aligned}
$$

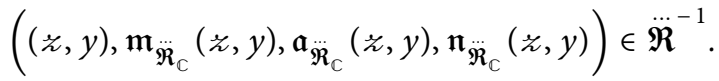

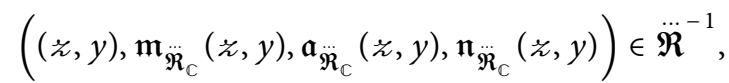$$
\left((y, x), \mathfrak{m}_{\mathfrak{R}_{\mathbb{C}}}(y, x), \mathfrak{a}_{\mathfrak{R}_{\mathbb{C}}}(y, x), \mathfrak{n}_{\mathfrak{R}_{\mathbb{C}}}(y, x)\right) \in \dddot{\mathfrak{R}}^{-1} .
$$

But $\dddot{R}$ is also a $\mathrm{CN}$ transitive relation. So

$$
\begin{aligned}
& \left((x, \approx), \mathfrak{m}_{\mathfrak{R}_{\mathbb{C}}}(x, \approx), \mathfrak{a}_{\mathfrak{R}_{\mathbb{C}}}(x, \approx), \mathfrak{n}_{\mathfrak{R}_{\mathbb{C}}}(x, \approx)\right) \in \dddot{R},
\end{aligned}
$$

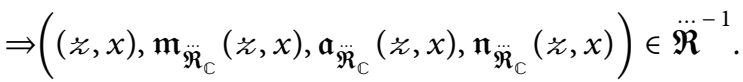

Hence, $\dddot{R}^{-1}$ is a $\mathrm{CN}$ transitive relation.

Thus by (45), (47), and (50), $\dddot{\mathfrak{R}}^{-1}$ is also a $\mathrm{CN}$-order relation. 
Definition 19. For a $\mathrm{CN}$ equivalence relation $\dddot{R}$ and $\left(x, \mathfrak{m}_{\ddot{A}_{C}}(x), \mathfrak{a}_{\ddot{A}_{C}}(x), \mathfrak{n}_{\ddot{A}_{C}}(x)\right) \in \ddot{A}$, the equivalence class of $x$ modulo $\dddot{R}$ is denoted and defined as

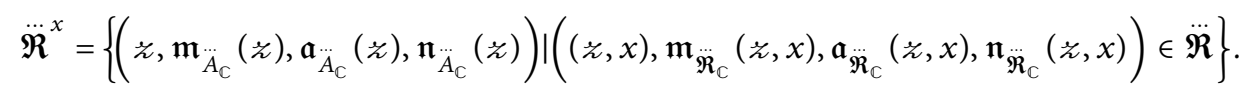

Example 9. For a $\mathrm{CN}$ equivalence relation,

$$
\dddot{R}=\left\{\begin{array}{c}
\left((x, x), 0.8 e^{(0.6) 2 \pi i}, 1 e^{(0.1) 2 \pi i}, 1 e^{(0.5) 2 \pi i}\right),\left((x, \approx), 0.3 e^{(0.5) 2 \pi i}, 0.5 e^{(0.1) 2 \pi i}, 1 e^{(0.5) 2 \pi i}\right), \\
\left((y, y), 0 e^{(0.8) 2 \pi i}, 0.9 e^{(0.4) 2 \pi i}, 0.5 e^{(1) 2 \pi i}\right),\left((\varkappa, x), 0.3 e^{(0.5) 2 \pi i}, 0.5 e^{(0.1) 2 \pi i}, 1 e^{(0.5) 2 \pi i}\right), \\
\left((\varkappa, \succsim), 0.3 e^{(0.5) 2 \pi i}, 0.5 e^{(0.6) 2 \pi i}, 0.7 e^{(0.2) 2 \pi i}\right)
\end{array}\right\} .
$$

The $\mathrm{CN}$ equivalence classes are

$$
\begin{aligned}
& \dddot{\mathfrak{R}}^{x}=\left\{\left(x, 0.8 e^{(0.6) 2 \pi i}, 1 e^{(0.1) 2 \pi i}, 1 e^{(0.5) 2 \pi i}\right),\right. \\
&\left.\cdot\left(\varkappa, 0.3 e^{(0.5) 2 \pi i}, 0.5 e^{(0.6) 2 \pi i}, 0.7 e^{(0.2) 2 \pi i}\right)\right\}, \\
& \dddot{\mathfrak{R}}^{y}=\left\{\left(y, 0 e^{(0.8) 2 \pi i}, 0.9 e^{(0.4) 2 \pi i}, 0.5 e^{(1) 2 \pi i}\right)\right\}, \\
& \dddot{\mathfrak{R}}^{*}=\left\{\left(x, 0.8 e^{(0.6) 2 \pi i}, 1 e^{(0.1) 2 \pi i}, 1 e^{(0.5) 2 \pi i}\right),\right. \\
&\left.\cdot\left(\varkappa, 0.3 e^{(0.5) 2 \pi i}, 0.5 e^{(0.6) 2 \pi i}, 0.7 e^{(0.2) 2 \pi i}\right)\right\} .
\end{aligned}
$$

Theorem 6. For a $C N$ equivalence relation $\dddot{R}$, $\left((x, y), \mathfrak{m}_{\dddot{R}_{\mathbb{C}}}(x, y), \mathfrak{a}_{\dddot{R}_{\mathbb{C}}}(x, y), \mathfrak{n}_{\mathfrak{R}_{C}}(x, y)\right) \in \dddot{\mathfrak{R}} \quad$ iff $\dddot{\mathfrak{R}}^{x}=$ $\dddot{\mathfrak{R}}^{y}$.

Proof. Suppose $\dddot{\mathfrak{R}}^{x}=\dddot{\mathfrak{R}}^{y}$, then for $\approx \in \dddot{\mathfrak{U}}$,

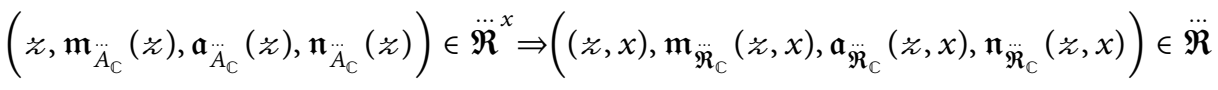

$$
\begin{aligned}
& \Rightarrow\left((x, \approx), \mathfrak{m}_{\mathfrak{R}_{C}}(x, \%), \mathfrak{a}_{\mathfrak{R}_{\mathbb{C}}}(x, \%), \mathfrak{n}_{\mathfrak{R}_{C}}(x, \%)\right) \in \dddot{\mathfrak{R}},
\end{aligned}
$$

because $\dddot{R}$ is a $\mathrm{CN}$ symmetric relation.

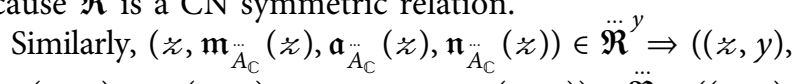
$\left.\mathfrak{m}_{\mathfrak{R}_{\mathbb{C}}}(\varkappa, y), \mathfrak{a}_{\mathfrak{R}_{\mathbb{C}}}(\varkappa, y), \mathfrak{n}_{\dddot{R}_{\mathbb{C}}} \quad(z, y)\right) \in \dddot{\mathfrak{R}} \Rightarrow((x, y)$, $\left.\mathfrak{m}_{\dddot{R}_{C}}(x, y), \mathfrak{a}_{\mathfrak{R}_{C}}(x, y), \mathfrak{n}_{\mathfrak{R}_{C}}(x, y)\right) \in \dddot{\mathfrak{R}}$ because $\dddot{\mathfrak{R}}$ is a CN transitive relation.

Conversely, suppose

$$
\begin{aligned}
& \left((x, y), \mathfrak{m}_{\mathfrak{R}_{\mathrm{C}}}(x, y), \mathfrak{a}_{\mathfrak{R}_{\mathrm{C}}}(x, y), \mathfrak{n}_{\mathfrak{R}_{\mathrm{C}}}(x, y)\right) \in \dddot{R}, \\
& \left(z, \mathfrak{m}_{\ddot{A}_{C}}(\approx), \mathfrak{a}_{\ddot{A}_{C}}(z), \mathfrak{n}_{\ddot{A}_{C}}(z)\right) \in \dddot{\mathfrak{R}}^{x} \text {, }
\end{aligned}
$$

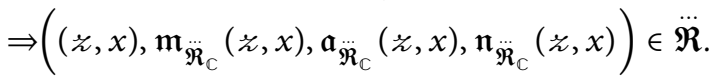

Then

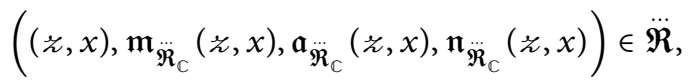

$$
\begin{aligned}
& \left((x, y), \mathfrak{m}_{\mathfrak{R}_{\mathbb{C}}}(x, y), \mathfrak{a}_{\mathfrak{R}_{\mathbb{C}}}(x, y), \mathfrak{n}_{\mathfrak{R}_{\mathbb{C}}}(x, y)\right) \in \dddot{R}, \\
& \Rightarrow\left((\varkappa, y), \mathfrak{m}_{\mathfrak{R}_{\mathrm{C}}}(\varkappa, y), \mathfrak{a}_{\mathfrak{R}_{\mathrm{C}}}(\varkappa, y), \mathfrak{n}_{\mathfrak{R}_{\mathbb{C}}}(\varkappa, y)\right) \in \dddot{R},
\end{aligned}
$$

because $\dddot{R}$ is a $\mathrm{CN}$ transitive relation

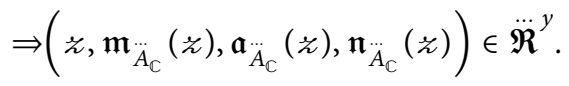


Hence,

$$
\dddot{\mathfrak{R}}^{x} \subseteq \dddot{\mathfrak{R}}^{y}
$$

Similarly, suppose

$$
\begin{aligned}
& \left((x, y), \mathfrak{m}_{\mathfrak{R}_{\mathrm{C}}}(x, y), \mathfrak{a}_{\mathfrak{R}_{\mathrm{C}}}(x, y), \mathfrak{n}_{\mathfrak{R}_{\mathbb{C}}}(x, y)\right) \in \dddot{\mathfrak{R}}, \\
& \left(\approx, \mathfrak{m}_{\ddot{A}_{\mathrm{C}}}(\approx), \mathfrak{a}_{\ddot{A}_{\mathrm{C}}}(\approx), \mathfrak{n}_{\ddot{A}_{\mathrm{C}}}(\approx)\right) \in \dddot{\mathfrak{R}}^{y} \text {, }
\end{aligned}
$$

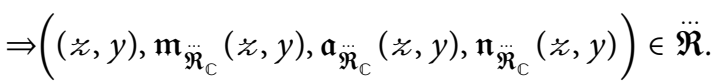

Since,

$$
\begin{aligned}
& \left((x, y), \mathfrak{m}_{\dddot{\mathfrak{R}}_{\mathbb{C}}}(x, y), \mathfrak{a}_{\mathfrak{R}_{\mathbb{C}}}(x, y), \mathfrak{n}_{\dddot{R}_{\mathbb{C}}}(x, y)\right) \in \dddot{\mathfrak{R}} \\
& \quad \Rightarrow\left((y, x), \mathfrak{m}_{\mathfrak{\Re}_{\mathbb{C}}}(y, x), \mathfrak{a}_{\mathfrak{R}_{\mathbb{C}}}(y, x), \mathfrak{n}_{\mathfrak{\Re}_{\mathbb{C}}}(y, x)\right) \in \dddot{\mathfrak{R}},
\end{aligned}
$$

because $\dddot{R}$ is a $\mathrm{CN}$ symmetric relation.

Now

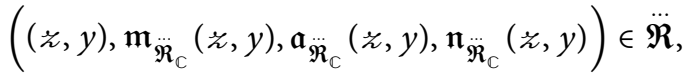

$$
\begin{aligned}
& \left((y, x), \mathfrak{m}_{\mathfrak{R}_{\mathbb{C}}}(y, x), \mathfrak{a}_{\mathfrak{R}_{\mathbb{C}}}(y, x), \mathfrak{n}_{\mathfrak{R}_{\mathbb{C}}}(y, x)\right) \in \dddot{\mathfrak{R}},
\end{aligned}
$$

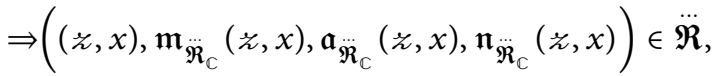

because $\dddot{R}$ is a $\mathrm{CN}$ transitive relation

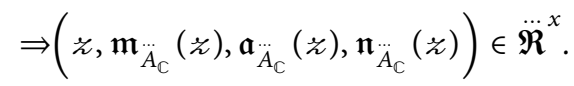

Hence,

$$
\dddot{\mathfrak{R}}^{y} \subseteq \dddot{\mathfrak{R}}^{x},
$$

(58) and (63) imply that $\dddot{R}^{x}=\dddot{\mathfrak{R}}^{x}$.

\section{Applications}

This section presents application of the proposed notions. It shows the worth of CNRs in decision-making for a statistician. The application uses the concept of CNRs in economic statistics and talks about the economic relationships.

Figure 1 depicts the economic factors whose relationships are discussed on the basis of statistical analysis in the following application.

4.1. Investigating the Economic Relationships Using Economic Statistics. Economics conveys the way businesses, governments, societies, and individuals allocate their wealth and resources. It also provides knowledge for making everyday decisions. The international financial affairs are concerned with the economics. The policies made by the financial ministers are thoroughly worked out before being applied because they can make or break the progress and economy of a nation. These economic policies are not just a shot in the dark but are properly calculated outcomes. These calculations are carried out by statisticians, who collect and organize the data, present data in the form of tables, diagrams, and graphs and then finally analyze and interpret the data. This process involves various statistical techniques at each stage, and Figure 2 summarizes each step.

An expert statistician carries out statistical analysis and makes some important decisions which are then implemented as the financial policies. The data at state levels are humongous and thus the probability of ambiguity, uncertainty, and imprecision is greater. So, CNSs and CNRs are worthwhile tools to cope with such kind of data and information. It will further improve the accuracy of the conclusions and thus help in making better decisions.

Let us consider a situation involving three economic factors, namely, investment (I), gross domestic product (GDP), and unemployment $(U)$. A decision-making statistician assigns each of the factors a degree of membership, a degree of abstinence, and a degree of nonmembership after going through the statistical process. In current supposition, the results of the experiment are assumed to be applicable for twelve months or a year at most.

Let

$$
\dddot{A}=\left\{\begin{array}{c}
\left(I, 0.8 e^{(0.75) 12 \pi i}, 0.2 e^{(0.5) 12 \pi i}, 0.2 e^{(0.25) 12 \pi i}\right),\left(\mathrm{GDP}, 1 e^{(1) 12 \pi i}, 0.4 e^{(0.5) 12 \pi i}, 0.3 e^{(0.16) 12 \pi i}\right), \\
\left(U, 0.4 e^{(1) 12 \pi i}, 0.5 e^{(0.25) 12 \pi i}, 0.1 e^{(0.08) 12 \pi i}\right)
\end{array}\right\} .
$$

Now, in order to find out the impacts of one factor on the other, a Cartesian product $\ddot{A} \times \dddot{A}$ needs to be found. Hence, 


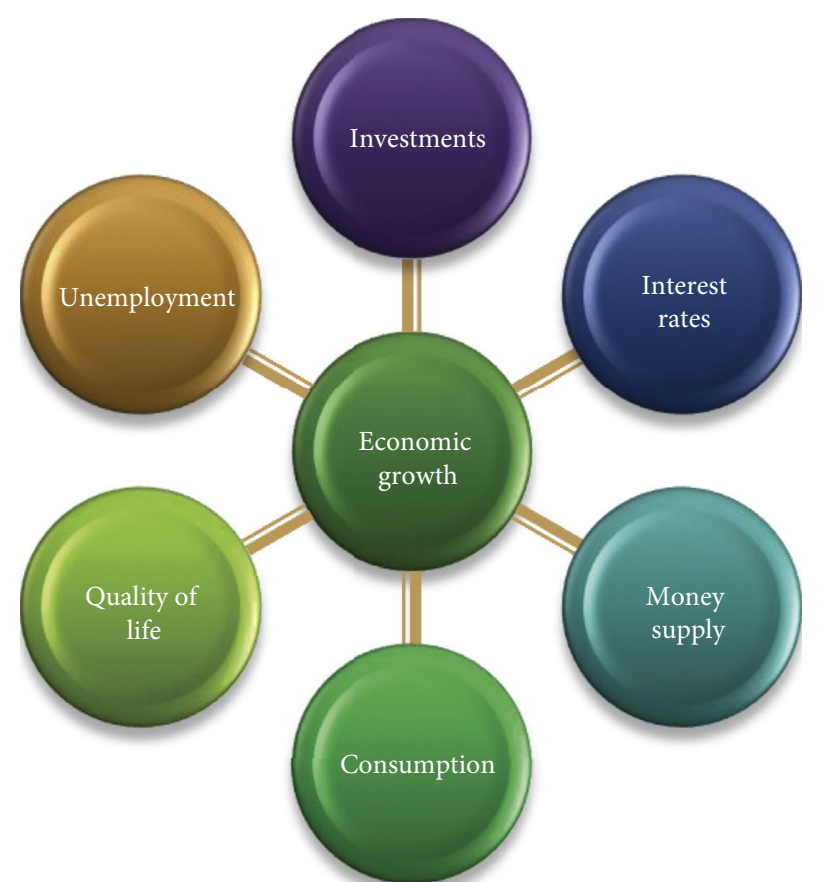

FIGURE 1: Factors revolving around the economic growth.

\begin{tabular}{ll} 
(i) arrays of data and tally bars \\
(i) tables, graphs, and diagrams \\
\hline
\end{tabular}

FIGURE 2: Statistical process for economic policy making.

$$
\dddot{A} \times \dddot{A}=\left\{\begin{array}{cc}
\left((I, I), 0.8 e^{(0.75) 12 \pi i}, 0.2 e^{(0.5) 12 \pi i}, 0.2 e^{(0.25) 12 \pi i}\right) & \left((\mathrm{GDP}), 0.8 e^{(0.75) 12 \pi i}, 0.2 e^{(0.5) 12 \pi i}, 0.3 e^{(0.25) 12 \pi i}\right) \\
\left((I, U), 0.4 e^{(0.75) 12 \pi i}, 0.2 e^{(0.25) 12 \pi i}, 0.2^{(0.25) 12 \pi i}\right) & \left.(\text { GDP, } I), 0.8 e^{(0.75) 12 \pi i}, 0.2 e^{(0.5) 12 \pi i}, 0.3 e^{(0.25) 12 \pi i}\right) \\
\left((\mathrm{GDP}, \mathrm{GDP}), 1 e^{(1) 12 \pi i}, 0.4 e^{(0.5) 12 \pi i}, 0.3 e^{(0.16) 12 \pi i}\right) & \left((\mathrm{GDP}, U), 0.4 e^{(1) 12 \pi i}, 0.4 e^{(0.25) 12 \pi i}, 0.3 e^{(0.16) 12 \pi i}\right) \\
\left((U, I), 0.4 e^{(0.75) 12 \pi i}, 0.2 e^{(0.25) 12 \pi i}\right) & \left((U, \mathrm{GDP}), 0.4 e^{(1) 12 \pi i}, 0.4 e^{(0.25) 12 \pi i}, 0.3 e^{(0.16) 12 \pi i}\right) \\
& \left((U, U), 0.4 e^{(1) 12 \pi i}, 0.5 e^{(0.25) 12 \pi i}, 0.1 e^{(0.08) 12 \pi i}\right)
\end{array}\right\} .
$$


Note that an ordered pair tells the impact of the first factor on the second one. Moreover, the degree of membership indicates the rate of influence. The degree of abstinence is taken to mean as the inability to decide that whether there is an influence of one factor on the other or not. And the degree of nonmembership indicates the rate of no influence. The amplitude term of each degree shows the strength of the impacts, and the phase terms refer to the time span in months. For example, the event $\left((I, U), 0.4 e^{(0.75) 12 \pi i}, 0.2 e^{(0.25) 12 \pi i}, 0.2 e^{(0.25) 12 \pi i}\right)$ expresses the impacts of investment $I$ on unemployment $U$. The numbers are translated in words as

(i) The degree of membership indicates the rate of influence of $I$ on $U$. In $0.4 e^{(0.75) 12 \pi i}, 0.4$ is the amplitude terms, and (0.75) 12 is the phase term. The translation is that influence of $I$ on $U$ is of degree 0.4 over the time span of 9 months, that is, very strong influence for a long duration. (ii) The degree of abstinence $0.2 e^{(0.25) 12 \pi i}$ interprets the inability to determine the existence of any influence is 0.2 over the time span of 3 months.

(iii) The degree of nonmembership $0.2 e^{(0.25) 12 \pi i}$ indicates that the degree of no influence is 0.2 over the period of 3 months.

Ignoring the $\mathrm{CN}$ reflexive relations and $\mathrm{CN}$ symmetric relations in (65), Table 1 shows the summary of all the impacts of one factor on the other.

Now an illustration of types of CNRs for a decision maker is proposed. It can reduce the efforts of a statistician in decision-making. Consider some other two CNSs such that set $\ddot{B}$ contains the currency value $(\mathrm{CV})$ and interest rate (IR) and set $\dddot{C}$ contains the dearness $(D)$ and quality of life (QL):

$$
\begin{aligned}
& \dddot{B}=\left\{\left(\mathrm{CV}, 0.6 e^{(0.66) 12 \pi i}, 0.4 e^{(0.25) 12 \pi i}, 0.6 e^{(0.08) 12 \pi i}\right),\left(\mathrm{IR}, 0.5 e^{(1) 12 \pi i}, 0.1 e^{(0.33) 12 \pi i}, 0.3 e^{(0.16) 12 \pi i}\right)\right\} \\
& \dddot{C}=\left\{\left(D, 0.4 e^{(0.75) 12 \pi i}, 0.8 e^{(0.25) 12 \pi i}, 0.5 e^{(0.08) 12 \pi i}\right),\left(\mathrm{QL}, 0.5 e^{(0.66) 12 \pi i}, 0.1 e^{(0.5) 12 \pi i}, 0.5 e^{(0.25) 12 \pi i}\right)\right\} .
\end{aligned}
$$

Finding the Cartesian products $\dddot{A} \times \dddot{B}$ and $\dddot{B} \times \dddot{C}$,

$$
\begin{gathered}
\dddot{A} \times \dddot{B}=\left\{\begin{array}{c}
\left((\mathrm{I}, \mathrm{CV}), 0.6 e^{(0.66) 12 \pi i}, 0.2 e^{(0.25) 12 \pi i}, 0.6 e^{(0.25) 12 \pi i}\right),\left((I, \mathrm{IR}), 0.5 e^{(0.75) 12 \pi i}, 0.1 e^{(0.33) 12 \pi i}, 0.3 e^{(0.25) 12 \pi i}\right), \\
\left((\mathrm{GDP}, \mathrm{CV}), 0.6 e^{(0.66) 12 \pi i}, 0.4 e^{(0.25) 12 \pi i}, 0.6 e^{(0.16) 12 \pi i}\right),\left((\mathrm{GDP}, \mathrm{IR}), 0.5 e^{(1) 12 \pi i}, 0.1 e^{(0.33) 12 \pi i}, 0.3 e^{(0.16) 12 \pi i}\right), \\
\left((\mathrm{U}, \mathrm{CV}), 0.4 e^{(0.66) 12 \pi i}, 0.4 e^{(0.25) 12 \pi i}, 0.6 e^{(0.08) 12 \pi i}\right),\left((\mathrm{U}, \mathrm{IR}), 0.4 e^{(1) 12 \pi i}, 0.1 e^{(0.25) 12 \pi i}, 0.3 e^{(0.16) 12 \pi i}\right)
\end{array}\right\} \\
\dddot{B} \times \dddot{C}=\left\{\begin{array}{c}
\left.(\mathrm{CV}, D), 0.4 e^{(0.66) 12 \pi i}, 0.4 e^{(0.25) 12 \pi i}, 0.6 e^{(0.08) 12 \pi i}\right),\left((\mathrm{CV}, \mathrm{QL}), 0.5 e^{(0.66) 12 \pi i}, 0.1 e^{(0.25) 12 \pi i}, 0.6 e^{(0.25) 12 \pi i}\right) \\
\left((\mathrm{IR}, D), 0.4 e^{(0.75) 12 \pi i}, 0.1 e^{(0.25) 12 \pi i}, 0.5 e^{(0.16) 12 \pi i}\right),\left((\mathrm{IR}, \mathrm{QL}), 0.5 e^{(0.66) 12 \pi i}, 0.1 e^{(0.33) 12 \pi i}, 0.5 e^{(0.25) 12 \pi i}\right)
\end{array}\right\} .
\end{gathered}
$$

Details of each of the relationships is given in Table 2 . Table 2 encapsulates the above relationships of $\dddot{A} \times \dddot{B}$ and $\dddot{B} \times \dddot{C}$, and the impacts of each factor on the other are described precisely with the time period.
The impacts of elements in set $\dddot{A}$ on the elements of set $\dddot{C}$ are easily found using the idea of $\mathrm{CN}$ composite relation. Let $\dddot{\mathfrak{R}}=(\dddot{A} \times \dddot{B}) \circ(\dddot{B} \times \dddot{C})$; then

$$
\dddot{R}=\left\{\begin{array}{c}
\left((I, D), 0.4 e^{(0.75) 12 \pi i}, 0.2 e^{(0.25) 12 \pi i}, 0.5 e^{(0.25) 12 \pi i}\right),\left((I, \mathrm{QL}), 0.5 e^{(0.66) 12 \pi i}, 0.1 e^{(0.5) 12 \pi i}, 0.5 e^{(0.25) 12 \pi i}\right) \\
\left((\mathrm{GDP}, D), 0.4 e^{(0.75) 12 \pi i}, 0.4 e^{(0.25) 12 \pi i}, 0.5 e^{(0.16) 12 \pi i}\right),\left((\mathrm{GDP}, \mathrm{QL}), 0.5 e^{(0.66) 12 \pi i}, 0.1 e^{(0.5) 12 \pi i}, 0.5 e^{(0.25) 12 \pi i}\right), \\
\left((U, D), 0.4 e^{(0.75) 12 \pi i}, 0.5 e^{(0.25) 12 \pi i}, 0.5 e^{(0.08) 12 \pi i}\right),\left((U, \mathrm{QL}), 0.4 e^{(0.66) 12 \pi i}, 0.1 e^{(0.25) 12 \pi i}, 0.5 e^{(0.25) 12 \pi i}\right)
\end{array}\right\}
$$

The above composite relation $\dddot{\Re}$ is completely discussed through Table 3.

Figure 3 portrays the impacts of all six factors on the investment $I$ or viceversa. The vertical $y$-axis represents the reading of phase terms when translated to months. The horizontal $x$-axis is labeled with numbers 1 to 6 , and each number represents a different factor that are mentioned just beneath the horizontal axis in the graph. Moreover, at each factor, there are three circles that represent the three degrees; green circles represent degree of membership, blue circles 
TABle 1: Details of impacts of one factor on the other in $\dddot{A} \times \dddot{A}$.

\begin{tabular}{lccc}
\hline Relation & $\begin{array}{c}\text { Rate of } \\
\text { influence }\end{array}$ & $\begin{array}{c}\text { Rate of } \\
\text { ambiguity }\end{array}$ & $\begin{array}{c}\text { Rate of no } \\
\text { influence }\end{array}$ \\
\hline$(I$, GDP $)$ & 0.8 in 9 months & 0.2 in 6 months & 0.3 in 3 months \\
$(I, U)$ & 0.4 in 9 months & 0.2 in 3 months & 0.2 in 3 months \\
$($ GDP, $U)$ & $\begin{array}{c}0.4 \text { in } 12 \\
\text { months }\end{array}$ & 0.4 in 3 months & 0.3 in 2 months \\
\hline
\end{tabular}

TAble 2: Details of impacts of each factor in $\dddot{A} \times \ddot{B}$ and $\dddot{B} \times \ddot{C}$.

\begin{tabular}{lccc}
\hline Relation & $\begin{array}{c}\text { Rate of } \\
\text { influence }\end{array}$ & $\begin{array}{c}\text { Rate of } \\
\text { ambiguity }\end{array}$ & $\begin{array}{c}\text { Rate of no } \\
\text { influence }\end{array}$ \\
\hline$(I, C V)$ & 0.6 in 8 months & 0.2 in 3 months & 0.6 in 3 months \\
$(I, I R)$ & 0.5 in 9 months & 0.1 in 4 months & 0.3 in 3 months \\
$(\mathrm{GDP}, \mathrm{CV})$ & 0.6 in 8 months & 0.4 in 3 months & 0.6 in 2 months \\
$(\mathrm{GDP}, \mathrm{IR})$ & $\begin{array}{c}0.5 \text { in } 12 \\
\text { months }\end{array}$ & 0.1 in 4 months & 0.3 in 2 months \\
$(U, \mathrm{CV})$ & 0.4 in 8 months & 0.4 in 8 months & 0.6 in a month \\
$(U, \mathrm{IR})$ & 0.4 in 12 & 0.1 in 4 months & 0.3 in 2 months \\
$(\mathrm{CV}, D)$ & 0.4 in 8 months & 0.4 in 3 months & 0.6 in a month \\
$(\mathrm{CV}, \mathrm{QL})$ & 0.5 in 8 months & 0.1 in 3 months & 0.6 in 3 months \\
$(\mathrm{IR}, D)$ & 0.4 in 9 months & 0.1 in 3 months & 0.5 in 2 months \\
$(\mathrm{IR}, \mathrm{QL})$ & 0.5 in 8 months & 0.1 in 4 months & 0.5 in 3 months \\
\hline
\end{tabular}

TABLE 3: Details of impacts of factors associated with $\mathfrak{R}$.

\begin{tabular}{lccc}
\hline Relation & $\begin{array}{c}\text { Rate of } \\
\text { influence }\end{array}$ & $\begin{array}{c}\text { Rate of } \\
\text { ambiguity }\end{array}$ & $\begin{array}{c}\text { Rate of no } \\
\text { influence }\end{array}$ \\
\hline$(I, D)$ & 0.4 in 9 months & 0.2 in 3 months & 0.5 in 3 months \\
$(I, Q L)$ & 0.5 in 8 months & 0.1 in 6 months & 0.5 in 3 months \\
$($ GDP,$D)$ & 0.4 in 9 months & 0.4 in 3 months & 0.5 in 2 months \\
$($ GDP, QL) & 0.5 in 8 months & 0.1 in 6 months & 0.5 in 3 months \\
$(U, D)$ & 0.4 in 9 months & 0.5 in 3 months & 0.5 in a month \\
$(U, Q L)$ & 0.4 in 8 months & 0.1 in 3 months & 0.5 in 3 months \\
\hline
\end{tabular}

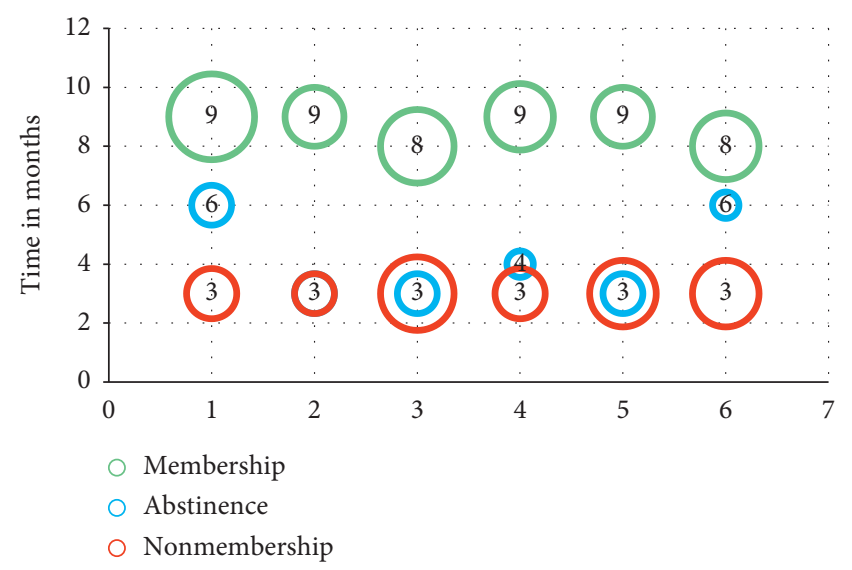

Figure 3: Impacts of investment $(I)$ on all other factors. The size of the circle indicates the degrees $\mathfrak{m}_{\mathbb{C}}, \mathfrak{a}_{\mathbb{C}}$, or $\mathfrak{n}_{\mathbb{C}}$. The numbers $1,2,3$, 4,5 , and 6 on the $x$-axis represent GDP, U, CV, IR, D, and QL, respectively. represent the degree of abstinence, and red circles represent the degree of nonmembership. Furthermore, the size of the circles indicates the value of the amplitude term, i.e., bigger circles means a higher values and smaller circles show a lower value of the amplitude term. The numbers inside the circles depict the time spans in months. Figures 4 and 5 illustrate the impacts of GDP and unemployment $U$ on the rest of the factors, respectively.

\section{Comparative Analysis}

This section compares the proposed methods with the existing ones such as FSs, CFSs, CIFSs, and their generalizations.

Since FSs, IFSs, and NSs cannot handle problems with periodicity, they are out of the picture in this race. It is convenient to carry out the comparison with CFSs, CIFSs, CPFSs, and CqROFSs because they are capable of dealing with multidimensional variables. In the above application, the relationships among the economic factors are studied. The decision makers are the expert economic statisticians, who collect, organize, and analyze the data before making any absolute decisions. The application discusses the usefulness of CNSs and CNRs in uncertain situations when dealing with large data. After carrying out the statistical analysis, the decision maker assigns three values to the parameters or factors. The degree of membership indicates the level of influence with respect to some time lag, the degree of abstinence interprets the inability to know if there is any influence or not for certain time, and the degree of nonmembership represents the level of no influence over some time interval. The CFSs are swept out of the competition as they only talk about the membership degree. While CIFSs discuss both the degrees of membership and nonmembership, the values are interdependent as their sum must be within the unit interval. This limitation restricts the decision maker, so he/she cannot efficiently make a better decision. As he/she is bound to choose numbers from certain limited sets. Like CIFSs, the CqROFSs are also characterized by the degrees of membership and nonmembership. The plus point of CqROFSs is that they do not have limitations in order to choose the values for the degrees of membership and nonmembership. But they still fail to solve the problem discussed above since they cannot indicate the degree of abstinence. As human opinion is not just restricted to yes or no, a CNS is a handy tool that can effectively cope with such issues. The CNSs are completely on another level because these sets allow the decision maker to freely assign any fuzzy number to each of the three degrees. Every degree is independent of the others. In the above application, the CIFSs, CPFSs, or CqROFSs could not have made better decisions as they do not discuss the abstinence degree. Moreover, in some cases, the sum of the degrees of membership and the degree of nonmembership exceeded 1. For instance, in $\left(\mathrm{CV}, 0.6 e^{(0.66) 12 \pi i}, 0.4 e^{(0.25) 12 \pi i}, 0.6 e^{(0.08) 12 \pi i}\right)$, the sum of amplitude terms in membership and nonmembership degrees is 1.2 which breaks the laws of CIFSs. Similarly, (GDP, $1 e^{(1) 12 \pi i}, 0.4 e^{(0.5) 12 \pi i}, 0.3 e^{(0.16) 12 \pi i}$ ) and $\left(U, 0.4 e^{(1) 12 \pi i}, 0.5 e^{(0.25) 12 \pi i}, 0.1 e^{(0.08) 12 \pi i}\right)$ are considered in a 


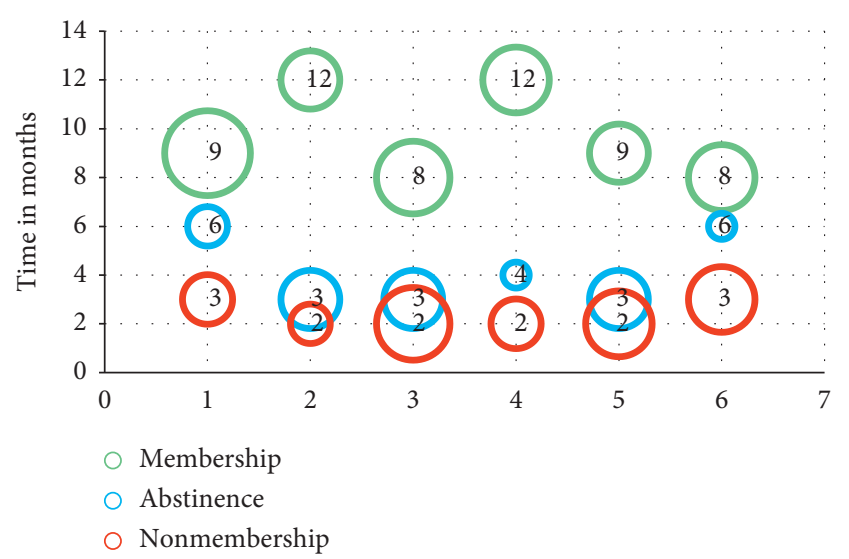

FIgURE 4: Impacts of GDP on all other factors. The size of the circle indicates the degrees $\mathfrak{m}_{\mathbb{C}}, \mathfrak{a}_{\mathbb{C}}$, or $\mathfrak{n}_{\mathbb{C}}$. The numbers $1,2,3,4,5$, and 6 on the $x$-axis represent I, $\mathrm{U}, \mathrm{CV}, \mathrm{IR}, \mathrm{D}$, and $\mathrm{QL}$, respectively.

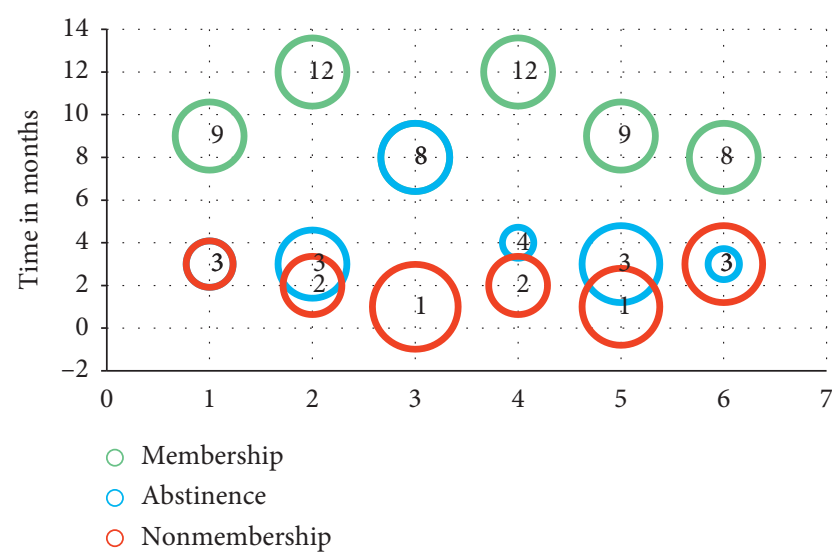

Figure 5: Impacts of unemployment $(U)$ on all other factors. The size of the circle indicates the degrees $\mathfrak{m}_{\mathbb{C}}, \mathfrak{a}_{\mathbb{C}}$, or $\mathfrak{n}_{\mathbb{C}}$. The numbers $1,2,3,4,5$, and 6 on the $x$-axis represent I, GDP, CV, IR, D, and QL, respectively.

CIFS because the sum of phase terms of both the degrees of membership and nonmembership exceed 1, i.e., $1+0.16 \not 1$ and $1+0.8 \nless 1$. Henceforth, NFSs and NFRs are superior tools to be applied in the above discussed scenario.

\section{Conclusion}

The main objective of this study was the introduction of the notion of CNRs which is a new concept. The CNSs and CNRs are characterized by three complex-valued mappings whose values range in the unit circle in a complex plane. The three mapping are the degrees of membership, abstinence, and nonmembership. Moreover, each degree consists of two different terms that are described by the real and imaginary parts of the complex numbers. The real part is called the amplitude term which represents the degree or level of membership, abstinence, or nonmembership, while the imaginary part is called the phase term that represents the time lag, periodicity, or phase changes in the degrees of membership, abstinence, or nonmembership. Furthermore, the types of CNRs are also introduced with suitable examples. In addition, the properties of CNRs are derived along with some useful results. An economic policy comes through many statistical procedures, and ultimately a decision is made on the basis of statistical analysis and outcomes. Henceforth, an application has been presented which emphasizes the utility of the proposed method in decisionmaking processes. Finally, a comparative analysis has been carried out among the existing methods and the proposed method.

\section{Data Availability}

No data were used to support the study.

\section{Conflicts of Interest}

The authors declare no conflicts of interest about the publication of the research article.

\section{Acknowledgments}

The authors are grateful to the Deanship of Scientific Research, King Saud University, for funding through Vice Deanship of Scientific Research Chairs.

\section{References}

[1] L. A. Zadeh, "Fuzzy sets," Information and Control, vol. 8, no. 3, pp. 338-353, 1965.

[2] K. T. Atanassov, "Intuitionistic fuzzy sets," Fuzzy Sets and Systems, vol. 20, no. 1, pp. 87-96, 1986.

[3] R. R. Yager, "Pythagorean fuzzy subsets," in Proceedings of the 2013 Joint IFSA World Congress and NAFIPS Annual Meeting (IFSA/NAFIPS), pp. 57-61, IEEE, Edmonton, Canada, June 2013.

[4] R. R. Yager, "Generalized orthopair fuzzy sets," IEEE Transactions on Fuzzy Systems, vol. 25, 2016.

[5] H. Garg, "Linguistic Pythagorean fuzzy sets and its applications in multiattribute decision-making process," International Journal of Intelligent Systems, vol. 33, no. 6, pp. 1234-1263, 2018.

[6] M. S. Yang and Z. Hussain, "Fuzzy entropy for Pythagorean fuzzy sets with application to multicriterion decision making," Complexity, vol. 2018, Article ID 2832839, 14 pages, 2018.

[7] Z. Hussain and M. S. Yang, "Distance and similarity measures of Pythagorean fuzzy sets based on Hausdorff metric with application to fuzzy TOPSIS," International Journal of Intelligent Systems, vol. 34, pp. 2633-2654, 2019.

[8] Q. Zhou, H. Mo, and Y. Deng, "A new divergence measure of pythagorean fuzzy sets based on belief function and its application in medical diagnosis," Mathematics, vol. 8, no. 1, p. 142, 2020.

[9] M. S. Yang, Z. Hussain, and M. Ali, "Belief and plausibility measures on intuitionistic fuzzy sets with construction of belief-plausibility TOPSIS," Complexity, vol. 2020, Article ID 7849686, 12 pages, 2020.

[10] S. Faizi, W. Sałabun, T. Rashid, S. Zafar, and J. Wątróbski, "Intuitionistic fuzzy sets in multi-criteria group decision making problems using the characteristic objects method," Symmetry, vol. 12, no. 9, p. 1382, 2020.

[11] X. Peng and L. Liu, "Information measures for $q$-rung orthopair fuzzy sets," International Journal of Intelligent Systems, vol. 34, no. 8, pp. 1795-1834, 2019. 
[12] G. Wei, H. Gao, and Y. Wei, "Some q-rung orthopair fuzzy Heronian mean operators in multiple attribute decision making," International Journal of Intelligent Systems, vol. 33, no. 7, pp. 1426-1458, 2018.

[13] D. Liu, X. Chen, and D. Peng, "Some cosine similarity measures and distance measures between $\mathrm{q}$-rung orthopair fuzzy sets," International Journal of Intelligent Systems, vol. 34, no. 7, pp. 1572-1587, 2019.

[14] F. Smarandache, "A unifying field in logics: neutrosophic logic," Neutrosophy, Neutrosophic Set, Neutrosophic Probability: Neutrsophic Logic. Neutrosophy, Neutrosophic Set, Neutrosophic Probability. Infinite Study, University of New Mexico Gallup, Gallup, NM, USA, 2005.

[15] H. Wang, F. Smarandache, Y. Zhang, and R. Sunderraman, "Single valued neutrosophic sets," Multispace and Multistructure, vol. 4, 2010.

[16] F. Smarandache, "Neutrosophic set-a generalization of the intuitionistic fuzzy set," Journal of Defense Resources Management (JoDRM), vol. 1, no. 1, pp. 107-116, 2010.

[17] F. Smarandache, "A geometric interpretation of the neutrosophic set-A generalization of the intuitionistic fuzzy set," 2004, http://arxiv.org/abs/0404520.

[18] F. Smarandache, "Neutrosophic set is a generalization of intuitionistic fuzzy set, inconsistent intuitionistic fuzzy set (picture fuzzy set, ternary fuzzy set), pythagorean fuzzy set, spherical fuzzy set, and q-rung orthopair fuzzy set, while neutrosophication is a generalization of regret theory, grey system theory, and three-ways decision (revisited)," Journal of New Theory, vol. 29, pp. 1-31, 2019.

[19] F. Smarandache, "Neutrosophic logic-a generalization of the intuitionistic fuzzy logic," Multispace \& Multistructure. Neutrosophic Transdisciplinarity (100 Collected Papers of Science), vol. 4, p. 396, 2010.

[20] F. Smarandache, "September. Definiton of neutrosophic logic-a generalization of the intuitionistic fuzzy logic," in Proceedings of the EUSFLAT Conference, pp. 141-146, Zittau, Germany, September 2003.

[21] A. A. Salama and S. A. Alblowi, "Neutrosophic set and neutrosophic topological spaces," IOSR Journal of Mathematics, vol. 3, no. 4, pp. 31-35, 2012.

[22] S. Das, B. K. Roy, M. B. Kar, S. Kar, and D. Pamučar, "Neutrosophic fuzzy set and its application in decision making," Journal of Ambient Intelligence and Humanized Computing, vol. 19, pp. 1-13, 2020.

[23] A. M. Khalil, D. Cao, A. Azzam, F. Smarandache, and W. R. Alharbi, "Combination of the single-valued neutrosophic fuzzy set and the soft set with applications in decision-making," Symmetry, vol. 12, no. 8, p. 1361, 2020.

[24] R. Şahin and P. Liu, "Correlation coefficient of single-valued neutrosophic hesitant fuzzy sets and its applications in decision making," Neural Computing and Applications, vol. 28, no. 6, pp. 1387-1395, 2017.

[25] R. M. Hashim, M. Gulistan, I. Rehman, N. Hassan, and A. M. Nasruddin, "Neutrosophic bipolar fuzzy set and its application in medicines preparations," Neutrosophic Sets Syst, vol. 31, pp. 86-100, 2020.

[26] D. Ramot, R. Milo, M. Friedman, and A. Kandel, "Complex fuzzy sets," IEEE Transactions on Fuzzy Systems, vol. 10, no. 2, pp. 171-186, 2002.

[27] A. S. Alkouri and A. R. Salleh, "Complex intuitionistic fuzzy sets," AIP Conference Proceeding, vol. 1482, p. 464, 2012.

[28] K. Ullah, T. Mahmood, Z. Ali, and N. Jan, "On some distance measures of complex Pythagorean fuzzy sets and their applications in pattern recognition," Complex \& Intelligent Systems, vol. 6, no. 1, pp. 15-27, 2019.
[29] P. Liu, T. Mahmood, and Z. Ali, "Complex Q-rung orthopair fuzzy aggregation operators and their applications in multi-attribute group decision making," Information, vol. 11, p. 5, 2020.

[30] L. Bi, S. Dai, B. Hu, and S. Li, "Complex fuzzy arithmetic aggregation operators," Journal of Intelligent \& Fuzzy Systems, vol. 36, no. 3, pp. 2765-2771, 2019.

[31] D. E. Tamir, N. D. Rishe, and A. Kandel, "Complex fuzzy sets and complex fuzzy logic an overview of theory and applications," Fifty Years of Fuzzy Logic and Its Applications, vol. 326, pp. 661-681, 2015.

[32] D. E. Tamir and A. Kandel, "Axiomatic theory of complex fuzzy logic and complex fuzzy classes," International Journal of Computers Communications \& Control, vol. 6, no. 3, pp. 562-576, 2011.

[33] J. Ma, G. Zhang, and J. Lu, "A method for multiple periodic factor prediction problems using complex fuzzy sets," IEEE Transactions on Fuzzy Systems, vol. 20, no. 1, pp. 32-45, 2011.

[34] R. T. Ngan, L. H. Son, M. Ali, D. E. Tamir, N. D. Rishe, and A. Kandel, "Representing complex intuitionistic fuzzy set by quaternion numbers and applications to decision making," Applied Soft Computing, vol. 87, p. 105961, 2020.

[35] H. Garg and D. Rani, "A robust correlation coefficient measure of complex intuitionistic fuzzy sets and their applications in decision-making," Applied Intelligence, vol. 49, no. 2, pp. 496-512, 2019.

[36] D. Rani and H. Garg, "Complex intuitionistic fuzzy power aggregation operators and their applications in multicriteria decision-making," Expert Systems, vol. 35, no. 6, p. e12325, 2018.

[37] Z. Ali and T. Mahmood, "Maclaurin symmetric mean operators and their applications in the environment of complex q-rung orthopair fuzzy sets," Computational and Applied Mathematics, vol. 39, pp. 1-27, 2020.

[38] P. Liu, M. Akram, and A. Sattar, "Extensions of prioritized weighted aggregation operators for decision-making under complex q-rung orthopair fuzzy information," Journal of Intelligent \& Fuzzy Systems, vol. 39, pp. 1-25, 2020.

[39] M. Ali and F. Smarandache, "Complex neutrosophic set," Neural Computing and Applications, vol. 28, no. 7, pp. 1817-1834, 2017.

[40] S. Broumi, A. Bakali, M. Talea et al., "Bipolar complex neutrosophic sets and its application in decision making problem," Fuzzy Multi-Criteria Decision-Making Using Neutrosophic Sets, 2019.

[41] M. Gulistan, A. Khan, A. Abdullahc, and N. Yaqoob, "Complex neutrosophic subsemigroups and ideals," International Journal of Analysis and Applications, vol. 16, no. 1, pp. 97-116, 2018.

[42] Z. Ali and T. Mahmood, "Complex neutrosophic generalised dice similarity measures and their application to decision making," CAAI Transactions on Intelligence Technology, vol. 5, no. 2, pp. 78-87, 2020.

[43] A. Al-Quran and N. Hassan, "The complex neutrosophic soft expert set and its application in decision making," Journal of Intelligent \& Fuzzy Systems, vol. 34, no. 1, pp. 569-582, 2018.

[44] S. Manna, T. M. Basu, and S. K. Mondal, "A soft set based VIKOR approach for some decision-making problems under complex neutrosophic environment," Engineering Applications of Artificial Intelligence, vol. 89, p. 103432, 2020.

[45] L. Q. Dat, N. T. Thong, L. H. Son et al., "Linguistic approaches to interval complex neutrosophic sets in decision making," IEEE Access, vol. 7, pp. 38902-38917, 2019.

[46] P. K. Singh, "Complex neutrosophic concept lattice and its applications to air quality analysis," Chaos, Solitons \& Fractals, vol. 109, pp. 206-213, 2018. 
[47] G. J. Klir and T. A. Folger, Fuzzy Sets, Uncertainty, and Information, Prentice-Hall, Englewood Cliffs, NJ, USA, 1988.

[48] J. M. Mendel, "Fuzzy logic systems for engineering: a tutorial," Proceedings of the IEEE, vol. 83, no. 3, pp. 345-377, 1995.

[49] P. Burillo and H. Bustince, "Intuitionistic fuzzy relations (Part I)," Mathware and Soft Computing, vol. 2, no. 1, pp. 5-38, 1995.

[50] P. A. Ejegwa, "Improved composite relation for Pythagorean fuzzy sets and its application to medical diagnosis," Granular Computing, vol. 5, no. 2, pp. 277-286, 2020.

[51] D. Ramot, M. Friedman, G. Langholz, and A. Kandel, "Complex fuzzy logic," IEEE Transactions on Fuzzy Systems, vol. 11, no. 4, pp. 450-461, 2003.

[52] B. Hu, L. Bi, S. Dai, and S. Li, "Distances of complex fuzzy sets and continuity of complex fuzzy operations," Journal of Intelligent \& Fuzzy Systems, vol. 35, no. 2, pp. 2247-2255, 2018.

[53] G. Deschrijver and E. E. Kerre, "On the composition of intuitionistic fuzzy relations," Fuzzy Sets and Systems, vol. 136, no. 3, pp. 333-361, 2003.

[54] H. Bustince and P. Burillo, "Structures on intuitionistic fuzzy relations," Fuzzy Sets and Systems, vol. 78, no. 3, pp. 293-303, 1996.

[55] H. Li, S. Yin, and Y. Yang, "Some preference relations based on q-rung orthopair fuzzy sets," International Journal of Intelligent Systems, vol. 34, no. 11, pp. 2920-2936, 2019.

[56] C. Zhang, H. Liao, and L. Luo, "Additive consistency-based priority-generating method of q -rung orthopair fuzzy preference relation," International Journal of Intelligent Systems, vol. 34, no. 9, pp. 2151-2176, 2019. 